\title{
植物叶片形态的生态功能、地理分布与成因
}

\author{
李耀琪 王志恒*
}

北京大学城市与环境学院生态研究中心, 地表过程分析与模拟教育部重点实验室, 北京 100871

\begin{abstract}
摘 要 叶片是植物与环境进行水气交换的重要场所, 形态多变。叶片形态可直接影响植物的生理生化过程，反映植物的资 源获取策略。该文以叶片大小、叶形、叶缘特征(有无叶齿)和叶型(单、复叶)等形态性状为例, 总结了当前叶片形态的研究 进展, 分析了叶形态性状的生态功能, 综述叶片形态的地理分布, 探讨叶片形态性状变化的驱动因素及其对生态系统功能的 影响。现有研究主要聚焦于局域尺度的特定类群, 关注叶大小、叶缘具齿性以及叶型的地理分布与生态成因, 发现叶片的形 态发育受基因调控, 叶形态性状与其他性状相互权衡, 其空间变异受气温和降水量共同驱动。以叶大小为代表的叶片形态性 状影响水分和养分循环，能够反映气候变化下的群落响应，也可用于预测生态系统初级生产力。今后应结合新方法获得覆盖 度高且区域无偏的数据, 探索叶形态在长时间尺度上的适应性进化, 研究叶形态特征及其对生态系统功能影响的尺度推绎。 该文有助于从叶片的角度认识植物对环境变化的响应, 以性状为桥梁将个体适合度、群落动态与生态系统功能联系起来, 能 够加深对植物群落生态学和功能生物地理学等相关领域研究进展的了解。
\end{abstract}

关键词 植物性状; 叶大小; 叶形; 叶型; 功能; 环境

李耀琪, 王志恒 (2021). 植物叶片形态的生态功能、地理分布与成因. 植物生态学报, 45, 1154-1172. DOI: 10.17521/cjpe.2020.0405

\section{Leaf morphological traits: ecological function, geographic distribution and drivers}

LI Yao-Qi and WANG Zhi-Heng*

Institute of Ecology, College of Urban and Environmental Sciences and Key Laboratory for Earth Surface Processes of the Ministry of Education, Peking University, Beijing 100871, China

\section{Abstract}

Leaf is one of the important organs of plants that facilitates the exchange of water and air with the surrounding environment. The morphological variation of leaves directly affect the physiological and biochemical processes of plants, which also reflects the adaptive strategies of plants to obtain resources. By focusing on several leaf morphological traits, including leaf size, leaf shape, leaf margin (with or without teeth) and leaf type (i.e. single vs. compound leaf), here, we reviewed the relevant research progresses in this field. We summarized the ecological functions of leaf morphological traits, identified their geographical distribution patterns, and explored the underlying environmental drivers, potential ecological interactions, and their effects on ecosystem functioning. We found that the current studies exploring the distribution and determinants of leaf size and leaf margin states mainly focused on single or specific taxon in local regions. Studies have also explored the genetic mechanisms of leaf morphology development. Leaf traits trade off with other functional traits, and their spatial variation is driven by both temperature and water availability. Leaf morphological traits, especially leaf size, influence water and nutrient cycling, reflect the response of communities to climate change, and can be scaled up to predict ecosystem primary productivity. Further studies should pay attention to combine new approaches to obtain unbiased data with high coverage, to explore the long-term adaptive evolution of leaf morphology, and to generalize the scaling in leaf morphology and its effect on ecosystem functioning. Leaf provides an important perspective to understand how plants respond and adapt to environmental changes. Studying leaf morphological traits provides insight into species fitness, community dynamics and ecosystem functioning, and also improves our understanding of the research progresses made in related fields, including plant community ecology and functional biogeography.

Key words plant traits; leaf size; leaf shape; leaf type; function; environment

Li YQ, Wang ZH (2021). Leaf morphological traits: ecological function, geographic distribution and drivers. Chinese Journal of Plant Ecology, 45, 1154-1172. DOI: 10.17521/cjpe.2020.0405 
叶片是植物进行光合作用和蒸腾作用的主要器 官, 是植物与外界进行水、气交换的重要门户，对生 态系统物质生产以及全球碳循环和水循环等均具有 重要影响(Chapin et al., 1993)。叶片形态多变, 表现 在其大小、形状、叶缘、叶尖、叶基和叶脉等多个 方面(图1)。基于此, 有学者提出自然界不存在两片 相同的叶子。叶形态是植物分类的重要指标, 研究 叶片的形态, 对于认识植物的系统进化, 了解植被 资源与分布，促进生物多样性保护具有重要意义。

叶片形态变化的生态和进化机制一直是植物学 和生态学领域研究的热点问题(Givnish, 1978a; Traiser et al., 2005; Peppe et al., 2014)。叶形态与植 物的生理-生物力学需求 (physiological and biomechanical demand)联系紧密(Feild et al., 2005; Royer \& Wilf, 2006; Peppe et al., 2011), 叶片形态的 变化是植物在选择压力下适应不同生境的一种表现,
反映了植物适应环境变化所形成的生存策略 (Baker-Brosh \& Peet, 1997; Vendramini et al., 2002; Liu et al., 2020)。例如, 大的叶片通常具有较高的光截获 能力(Niinemets et al., 2005; Niklas et al., 2007; Smith et al., 2017; Lusk et al., 2019), 又因边界层较厚、边 界层导度较低、叶表温度较高, 而提高了光合和暗 呼吸速率(Mebrahtu et al., 1991; Okajima et al., 2012)。小叶具有较高的主脉密度和较高的水力导度, 可抵抗干旱(Scoffoni et al., 2011; Sack et al., 2012)。 叶齿通常被认为是对寒冷的适应, 能通过增强液流, 促进叶片与外界的气体交换和碳固定 (Royer \& Wilf, 2006; Peppe et al., 2011), 还可通过 吐水作用释放叶肉细胞间过多的水流(mesophyll flooding), 缓解过高的根压(Feild et al., 2005)。叶片 形态发育受多种遗传因素影响, 如激素、转录因子、 微核糖核酸、小分子肽和外基因标记等, 通过诱导

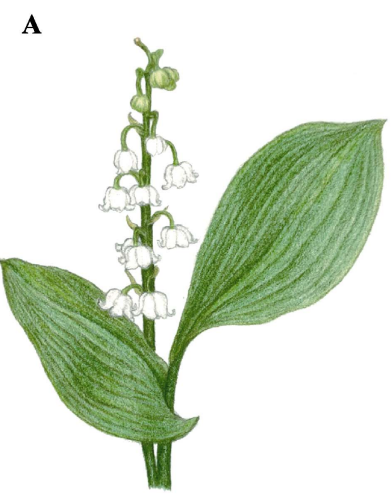

D

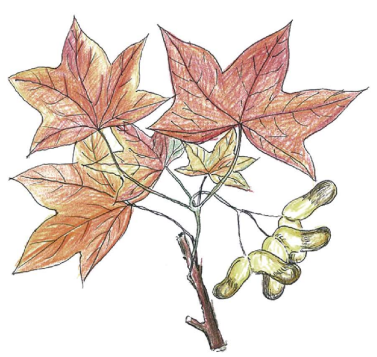

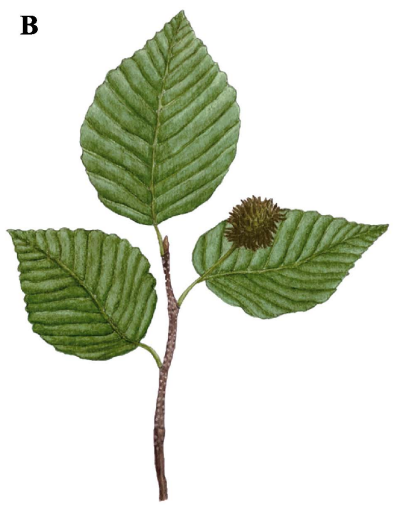

$\mathbf{E}$

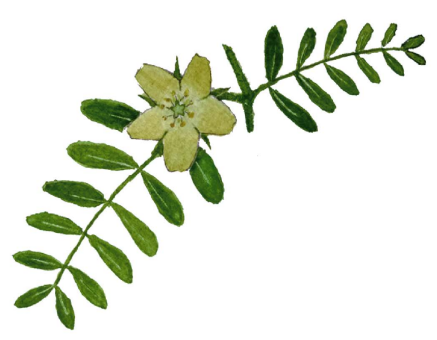

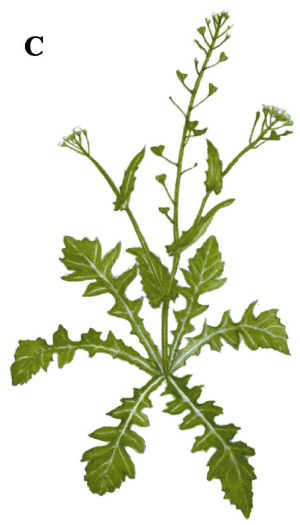

F

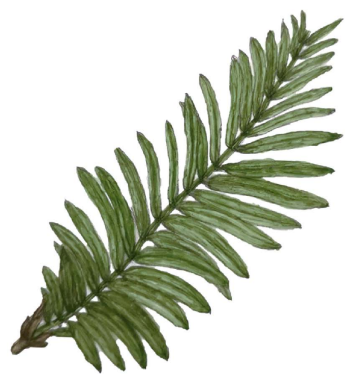

图1 不同形态叶片的手绘图。 $\mathbf{A}$, 铃兰, 单叶, 全缘, 叶椭圆形或卵状披针形。 $\mathbf{B}$, 水青冈, 单叶, 叶缘有锯齿, 叶卵形或长 卵形。C, 荠, 基生叶丛生呈莲座状, 大头羽状分裂, 顶裂片卵形至长圆形, 侧裂片长圆形至卵形; 茎生叶抱茎, 窄披针形或披 针形, 边缘有缺刻或锯齿。D, 元宝枫, 单叶, 掌状 5 裂。E, 䟻䒩, 偶数羽状复叶, 小叶矩圆形或斜短圆形。F, 水杉, 叶条形, 呈 羽状排列。A-C, F, 蔡琼绘; D, 买一慧绘; E, 罗晓图绘。描述参考中国植物志中英文版(http://www.efloras.org/flora_page. aspx?flora id=2)。

Fig. 1 Leaves with varied morphology. A, Convallaria majalis, single leaf with entire margin, leaf blade elliptic to ovate-lanceolate. B, Fagus longipetiolata, single leaf with serrate margin, blade ovate to ovate-oblong. C, Capsella bursa-pastoris, basal leaves rosulate, leaf blade oblong or oblanceolate; cauline leaves amplexicaul, narrowly oblong, lanceolate, or linear, margin entire or dentate. D, Acer truncatum, single leaf, usually 5-lobed. E, Tribulus terrestris, even-pinnately compound leaf, leaflet blades oblong to obliquely oblong, margin entire. F, Metasequoia glyptostroboides, linear leaves, pinnately aligned. Hand painted by CAI Qiong (A-C, F), MAI Yi-Hui (D), and LUO Xiao-Tu (E). Leaf morphology description was obtained from eflora (http://www.efloras.org/flora_page. aspx?flora_id=2). 
叶原基、建立背腹性及调控叶片扁平化等过程, 进 而形成不同大小和形状的叶片(Ali et al., 2020; Ren et al., 2020)。例如, 近期研究证明, TCP转录因子在 植物叶形态多样化的过程中起到重要作用, 其与转 录抑制因子TIE1和E3泛素连接酶TEAR1相互作用, 共同调控植物的叶形态(Tao et al., 2013; Zhang et al., 2017)。

目前叶形态的研究主要集中于两个方面。一方 面是叶性状间的权衡关系, 主要研究叶片形态、结 构和生理性状间的关系, 包括叶片大小与数目间的 权衡(Kleiman \& Aarssen, 2007; Whitman \& Aarssen, 2009; Huang et al., 2016)、叶形态对叶片生理结构 (如叶脉网络、栅栏组织和海绵组织)和支撑结构(如 小枝、叶柄)间生物量分配的影响(Milla \& Reich, 2007; Niinemets et al., 2007; Yang et al., 2010; Tian et al., 2016; He et al., 2018)以及叶片大小和排列对 叶片光合作用、水分运输等生理过程的影响(Poorter \& Rozendaal, 2008; Pyakurel \& Wang, 2014)。另一方 面是叶形态与环境间的关系及其应用。以往研究发 现, 多个叶形态指标(包括叶片大小、叶片长宽比、 叶缘类型、单复叶等)与环境因子(主要为年平均气 温、降水量和土壤性质)间具有较强的相关性(Jacobs, 1999; Royer et al., 2005; Traiser et al., 2005; Adams et al., 2008; Xia et al., 2009; Peppe et al., 2011)。例如, 在干旱、寒冷或盐碱环境下, 群落中的植物以小叶 或肉质叶为主, 而在湿热环境下大叶植物较多 (McDonald et al., 2003; Peppe et al., 2011); 在寒冷 气候下叶片边缘通常具有较大、较多的齿, 且叶片 倾向于高度分裂(Peppe et al., 2011)。此外, 叶形态 对气候变化响应敏感(Wiemann et al., 1998; Royer et al., 2009b; Guerin et al., 2012; Li et al., 2020b)。因 此, 叶形态的变异能有效反映生境的变化, 常用来 重建古气候(Wilf et al., 1998; Greenwood et al., 2004; Adams et al., 2008; Peppe et al., 2011; Chen et al., 2014)。定量叶形态与气候间的关系有助于理解植物 对气候变化的响应(Dolph \& Dilcher, 1980; Fonseca et al., 2000; Peppe et al., 2011), 亦可用来推测全球 气候变化对植被组成和分布的影响(Kowalski, 2002; Yang et al., 2016, 2019b), 为植被-气候模型的构建 提供新的视角。

可见, 叶片形态作为植物对生境的一种适应策 略, 不同的叶形态直接影响着植物的生理生化过程,
叶形态的变异亦能有效地反映生境的变化 (McDonald et al., 2003; Peppe et al., 2011)。研究叶形 态变异的生态和进化机制以及叶形态变化对生态系 统功能的影响一直是植物生态学研究的热点。本文 通过回顾研究进展, 总结叶形态(包括叶大小、叶 形、叶缘和叶型等)的生态功能, 综述叶形态的地理 分布, 分析叶形态变化的驱动因子, 从叶片的角度 认识植物对外界环境的生态适应及其对生态系统功 能的影响, 以期促进相关领域的研究。

\section{1 叶片大小的生态功能及其地理格局}

\section{1 叶片大小的生态功能}

叶片的大小是叶形态研究中广受关注的性状。 研究叶大小最常用的指标是叶片表面积。早期研究 常利用分级的方法定性估算叶面积大小, 常用的叶 面积分级估算方法有 Raunkiaer-Webb系统 (Webb, 1959) 和气候 - 叶相多变量综合分析项目 (Climate-Leaf Analysis Multivariate Program, CLAMP) (Wolfe，1993), 但分级定性估算方法无法准确估算 某物种或地区的叶面积大小。近年来，人们更多采 用定量测量方法估算叶面积, 主要方法有: 方格 法、称纸重法、打孔称重法、回归方程法和数字图 像处理技术法等(热娜古丽-热西提等, 2020)。前三 种方法相对比较精确, 但工作量大, 适用于样本量 较少的情况。此外, 这些方法易受叶片脱水萎缩的 影响, 且其中的打孔法属于破坏性测量。回归方程 法一般是基于叶长、叶宽与叶面积间的回归关系估 算叶面积。多个基于单物种的研究指出, 叶面积与 叶长和叶宽的乘积呈显著的线性关系(Cristofori et al., 2007; Rouphael et al., 2010)。植物志中通常收录 有物种叶长和叶宽的范围, 可用于批量估算叶面 积。图像处理方法是目前叶面积测量的主要方法, 在野外采集新鲜叶片后先进行扫描存储, 后续再用 专门的图像处理软件(例如ImageJ)根据比例尺和像 元数估算叶面积。该方法需要注意保持叶片湿润平 展, 避免脱水萎蒸。

叶大小对植物的生理过程具有多方面的影响。 首先, 叶大小反映了植物对光的截获能力。基于种 群或同一区域中多个物种的研究指出, 光获取效率 随叶面积增大而增大, 大叶具有较高的光获取效率 (Niinemets et al., 2005; Niklas et al., 2007; Smith et al., 2017; Lusk et al., 2019)。这可能因为以下几点:

www.plant-ecology.com 
1)大叶通常具有较长的叶柄, 且出叶强度 (leafing intensity)较低(Wang et al., 2019), 叶片在空间上排 列分散, 即单位小枝上叶数较少(Duursma et al., 2012; Smith et al., 2017), 因此叶片间遮蔽程度 (self-shading)较低(Falster \& Westoby, 2003), 光获取 面积较大；2) 随着叶面积增大, 叶绿素 $\mathrm{a} / \mathrm{b}$ 增大 (Terashima \& Hikosaka, 1995), 补光能力增强; 3)大 叶的扁平细胞占比大, 气孔密度较低(Conesa et al., 2020), 主脉密度也较低(Scoffoni et al., 2011), 叶内 遮阴可能较弱, 与小叶相比, 对到达叶绿体的光照 量限制要小(Terashima \& Hikosaka, 1995)。因此, 不 考虑叶倾角、叶排列以及风速等外界条件, 大叶通 常具有更大的光截获效率或光截获能力, 可显著影 响植物的光合生产能力 (Terashima \& Hikosaka, 1995; Duursma et al., 2012; Lusk et al., 2019)。其次, 叶大小可通过限制叶片表面气孔的数目和叶脉分布 间接影响植物的水分利用效率。有研究指出, 叶大 小与一些水分利用相关的植物性状密切相关, 包括 叶脉密度(Price et al., 2012; Sack et al., 2012)、气孔 密度(Pyakurel \& Wang, 2014; Conesa et al., 2020)、比 叶面积(Fonseca et al., 2000; Ackerly et al., 2002; Milla \& Reich, 2007)、树高以及木材密度(Malhado et al., 2009)等, 进而影响叶片乃至整个植株的生理活 动。例如, 多个研究指出主脉密度与叶大小呈负相 关关系, 而细脉密度相对独立(Scoffoni et al., 2011; Sack et al., 2012; Kawai \& Okada, 2016; Schneider et al., 2017), 二者共同决定了叶片的水力导度和水分 供应容量(Scoffoni et al., 2011; Schneider et al., 2017)。通过分析全球485种双子叶植物的叶脉结构 与叶大小的关系, Sack等(2012)发现, 大的叶片具有 较大直径的主脉, 但主脉密度较低。小叶因较高的 主脉密度, 具有较高的水力导度和较低的干旱脆弱 性(Scoffoni et al., 2011; Nardini et al., 2014)。又如, 气孔密度随着叶面积减小而增大(Franks \& Farquhar, 2007; Peel et al., 2017; Conesa et al., 2020), 决定了 叶片的水分损失和最大光合速率(Schneider et al., 2017)。最新的研究结合同质园(common garden)实验 和贝叶斯多层模型指出, 叶面积与气孔密度显著负 相关, 但与气孔长度不相关(Conesa et al., 2020)。这 可能是因为大叶具有较大比例的扁平细胞, 因而气 孔密度较低(Conesa et al., 2020), 而小叶具有较高 的细胞嵌入度(Salisbury \& Oliver, 1928), 高气孔密
度可满足光合所需的 $\mathrm{CO}_{2}$ 通量(Franks \& Farquhar, 2007)。第三, 叶大小可显著影响叶片的能量平衡(辐 射增温和蒸腾降温)。随叶片增大, 边界层厚度增大, 边界层导度降低(Schuepp, 1993; Wright et al., 2017), 叶片内 $\mathrm{CO}_{2}$ 浓度降低, 因此叶表与空气间的温度差 ( $\Delta T$ )增大(Nobel, 2009; Okajima et al., 2012; Wright et al., 2017)。大叶温度较高, 可补偿叶片表面降低 的 $\mathrm{CO}_{2}$ 含量, 以提高光合和暗呼吸速率(Mebrahtu et al., 1991; Okajima et al., 2012)。当风速可忽略时, 叶 表温度与叶大小呈正相关关系(Schuepp, 1993; Yates et al., 2010; Okajima et al., 2012; Wright et al., 2017), 进而影响叶片的生理、生化过程以适应外界气候的 变化。

在自然条件下, 生活在不同生境的植物通常具 有不同的叶大小。叶大小的变化可能受多种因素影 响。首先, 叶大小的变化可能反映了植物在最大化 光合生产能力和适应逆境的权衡。不同大小的叶片 通过调节叶片边缘处的空气对流速度影响叶片表面 温度, 以最大化碳吸收能力并提高水分利用效率 (Parkhurst \& Loucks, 1972; Michaletz et al., 2016)。对 于大叶植物, 随着温度的升高, 光合作用所增加的 碳收益可能会低于因蒸滕作用加快而造成的水分散 失(Givnish \& Vermeij, 1976; Givnish, 1984; Fonseca et al., 2000), 因此大叶仅在水分充足的温暖地区是 有利性状。在强辐射、炎热且干旱的地区, 大叶因 边界层较厚, 叶片表面与空气间温度差增大, 白天 辐射增温大于蒸腾冷却, 叶片容易热损伤; 而小叶 则因较薄的边界层, 不易过热损伤, 拥有更接近于 光合作用的最适叶温, 光合速率加快(Scoffoni et al., 2011; Okajima et al., 2012), 同时小叶具有较低的气 孔导度, 蒸腾速率降低, 可实现水分利用效率的最 大化(Parkhurst \& Loucks, 1972; Okajima et al., 2012)。在寒冷的夜间, 大叶又因边界层导度低而阻 碍感热通量, 叶温低于空气温度, 容易受夜间冻害 (Schuepp, 1993; Wright et al., 2017)。因此, 与大叶相 比, 小叶因较高的水分利用效率且不易受热损伤或 夜间冻害, 是对强辐射、炎热且干旱的环境或者寒 冷环境的适应(Scoffoni et al., 2011; Okajima et al., 2012; Leigh et al., 2017; Wright et al., 2017)。其次, 叶大小反映了植物不同组织结构间的生物量分配。 通过整合2 000 余种植物的性状数据, 研究发现叶面 积是冠层高度、种子质量和叶干质量的常数函数, 
即叶大小与植物整株和种子大小间存在异速增长关 系, 虽然在干扰或者贫㾑情况下有例外(Hodgson et al., 2017)。研究指出, 比叶面积随着叶片增大而减 小, 表明大叶子虽然有较大的光截获面积, 但单位 叶面积所需的干物质投入更大, 意味着叶子获取光 能的代价增大(Niinemets, 2001; Milla \& Reich, 2007; Niklas et al., 2007)。叶大小与比叶面积的异速增长 关系可能与叶片在叶柄和中脉等支撑性结构上的投 入有关(Niinemets et al., 2007)。与小叶相比, 大叶脉 管和厚壁细胞增多以保证水分供应和养分运输, 也 需要更长的叶柄以减少叶片间的自荫作用, 因此大 叶需要分配更多的干物质用于支撑结构(Milla \& Reich, 2007; Niinemets et al., 2007; Yang et al., 2010)。依据科纳法则(Corner's rule), 小枝的大小与 小枝所承受的附加物(如叶柄、叶片)大小呈正相关 关系(Corner, 1949; Smith et al., 2017)。对中国四川4 个阔叶林 234 种植物的研究发现, 叶面积与茎和小 枝的质量呈异速增长的关系, 且这种关系不受植物 生活型和生境类型的影响(Yang et al., 2010)。此外, 叶大小与叶片的解剖、生化性状紧密相关。例如, 叶 大小影响叶脉分布和主脉密度, 一般而言, 主脉和 侧脉(即一级和二级叶脉)的密度随着叶大小的增大 而呈几何递减(Sack et al., 2012; Schneider et al., 2017); 大叶多具有较密的表皮毛和稀疏的气孔以 减少叶片的水分损失(Pyakurel \& Wang, 2014), 小 叶具有较大的气孔密度和中脉厚度, 以降低呼吸速 率, 改善光合潜力(Liu et al., 2020)。综上, 叶大小反 映了植物的资源获取策略, 是长期进化过程中对环 境梯度的适应和投入-产出的权衡, 受温度和水分 共同限制, 也与不同组织器官间的生物量分配和叶 片结构性状等有关。

以往研究指出, 功能性状的变异受进化历史和 环境变化的共同驱动, 反映了植物资源获取策略和 对环境的适应性变异(Reich et al., 2003)。宏观大尺 度上, 叶大小更多地受气候而非进化历史的影响。 基于中国 1 万余种木本植物的分析发现, 叶大小在 科、属水平上均未表现出显著的谱系信号, 表明叶 大小谱系不保守, 在进化上不稳定(Li et al., 2020a)。 叶大小作为一种谱系不保守的性状, 其变异更多地 受持续的环境筛选而非进化历史的影响(Reich et al., 2003; Schellenberger Costa et al., 2018)。对中亚干旱 区典型的荒漠灌木一一红砂(Reaumuria soongarica)
野生类群的研究表明, 红砂倾向于改变叶长以应对 环境变化; 相应地，对比野外与同质园的类群发现， 叶宽的变化是局域环境和表型可塑性共同作用的结 果(Fan et al., 2020)。基于Populus tremuloides 492 个 基因型的同质园实验发现，虽然叶大小也受基因型 影响, 树冠位置对叶大小变异的影响要远高于基因 型(Eisenring et al., 2021)。因此, 叶大小主要受光、 叶片表面温度、水分可利用性等非生物因素的影响, 也受光竞争、取食防御等生物因素的限制(Reich et al., 2003; Williams et al., 2020)。

叶大小对气候变化的响应敏感，可作为生境气 候的指示因子(Werger \& Ellenbroek, 1978; Greenwood, 1992; Malhado et al., 2009; Guerin et al., 2012; Li et al., 2020b)。例如, Werger和Ellenbroek (1978)通 过研究南非奥兰治河沿岸森林中木本植物的叶大小 发现, 当气候从温暖变为炎热、干旱时, 叶片大小发 生分化，小型叶被更小的叶片取代，大型叶消失。 $\mathrm{Li}$ 等(2020b)基于中国自1910年以来的6 000余份叶 标本发现, 叶大小的年际变化与降水量变化呈显著 正相关关系。此外, Wilf等(1998)通过整合50个森林 样点发现, 植物叶面积与降水量有很强的相关性, 指出该关系可用来重建更新世降水格局。

\section{2 叶大小的空间格局及其主导因子}

目前对叶片大小空间分布的研究多集中在某些 类群沿海拔的分布(Milla \& Reich, 2011; Li \& Bao, 2014; Liu et al., 2020)以及多类群沿纬度的分布，主 要有Traiser等(2005)对欧洲阔叶乔木叶大小的分布 研究，Li等(2020a)对中国和北美木本植物叶大小的 研究(图2), Wright等(2017)对全球叶大小的研究。对 欧洲阔叶乔木的研究发现, 小型叶主要分布在地中 海沿岸, 大型叶在地中海沿岸和北方内陆分布均较 多(Traiser et al., 2005)。对中国木本植物的研究发现, 叶片平均大小从中国东南到西北逐渐减小(Li et al., 2020a)。

叶大小的空间分布主要受气候驱动，在温暖、 降水充沛且没有直接强光辐射的地区, 植物一般具 有较大的叶片, 而在寒冷、光辐射较强、干旱或土 壤贫㾑的地方, 植物通常具有较小的叶片(Dolph \& Dilcher, 1980; Ackerly et al., 2002; Xu et al., 2009; Wright et al., 2017), 以避免叶片过热或减少水分损 失(Okajima et al., 2012; Leigh et al., 2017; Wang et al., 2019)。在群落水平上, 平均叶大小随降水量 

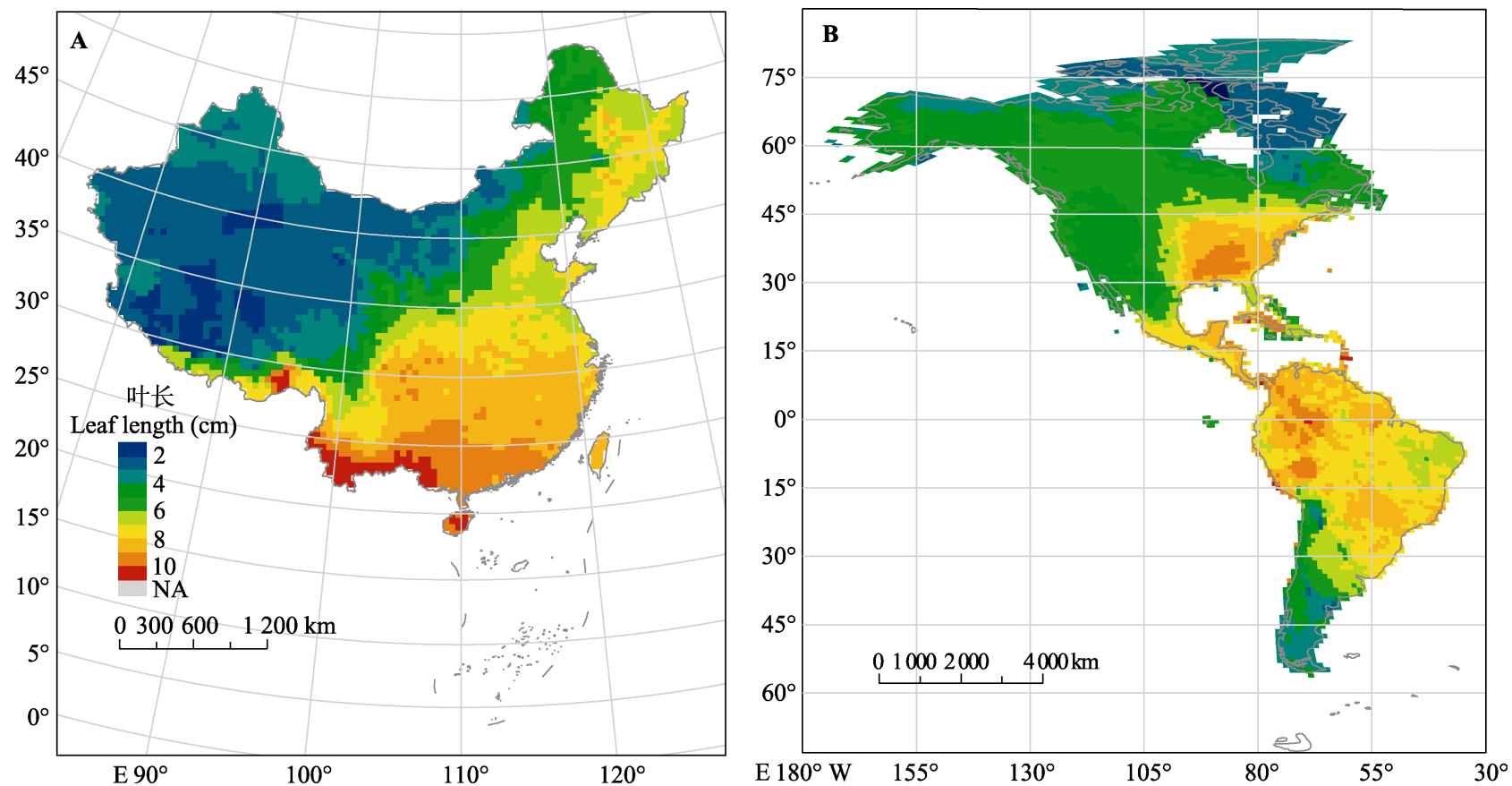

图2 中国和新世界(南、北美洲)木本植物平均叶长的地理格局。改编自Li等(2020a)附录图S1.1a和S6.1a。NA, 空值。

Fig. 2 Geographic patterns of leaf length for woody plants in China and in New World (i.e. North and South America). Modified from the appendices S1.1a \& S6.1a in Li et al. (2020a). NA, not available.

增多、气温升高而变大(Li et al., 2020a)。这是因为 干旱环境下, 小叶可减少蒸腾作用引起的水分、热 量损失, 而当水分不是限制因素时, 增大有效光合 叶面积才是对植物生长更有利的策略(Parkhurst \& Loucks, 1972; Givnish, 1984; Okajima et al., 2012)。 除了气温和降水量等气候因子, 辐射、光和风速对 叶大小也有一定的影响(Ackerly et al., 2002; Li et al., 2020a; Williams et al., 2020)。Ackerly等(2002)通过 研究地中海22种浓密常绿阔叶灌从灌木(chaparral shrub)的叶大小沿海拔和坡向的分布发现, 大叶植 物在不同辐射梯度上均有分布, 但小叶植物集中分 布在辐射较强的南坡。基于同质园 37 个种群的分析 指出, 光强解释了叶大小等性状在种内的大部分变 异(Williams et al., 2020)。此外, 叶大小也受树冠高 度/位置的影响, 如叶面积一般沿着树冠从低处到 高处递增(Jahdi et al., 2020; Eisenring et al., 2021)。 受资金、时间等成本影响, 先前研究多关注特定类 群, 取样数量有限; 基于部分类群的叶大小分析是 否能代表当地整体情况还需进一步探索。

目前, 叶大小的气候主导因子或者说降水量和 气温对叶大小空间变异的相对作用强弱还存在一定 争议 (Greenwood, 1992; McDonald et al., 2003; Traiser et al., 2005; Li \& Bao, 2014)。例如, 有研究指 出大叶物种比例与降水量有较弱的负相关性(Werger
\& Ellenbroek, 1978; Malhado et al., 2009), 但基于中 国木本植物群落的研究发现, 平均叶大小与降水量 呈较强的正相关关系(Li et al., 2020a)。又如, 对南北 美洲热带地区叶片的研究发现, 叶大小对降水量的 响应比对温度更敏感(Dolph \& Dilcher, 1980; Ordoñez et al., 2009), 但有整合分析表明, 包括叶大小 在内的大多数植物性状与年平均气温间的关系均显 著强于其与年降水量间的关系(Moles et al., 2014)。 最近关于全球叶大小分布的研究指出, 叶大小在寒 冷地区与年降水量间关系不显著, 但在温暖地区与 年降水量呈正相关关系, 且这种关系随气温的增加 而增强, 暗示了叶大小受水热交互作用的影响 (Wright et al., 2017)。综上, 叶大小显著受气温、降 水量及其季节性(seasonality)的影响(Dolph \& Dilcher, 1980; Ackerly et al., 2002; Wright et al., 2017)。

\section{3 叶大小与生态系统功能}

叶大小反映了叶片的资源获取能力, 尤其是光 获取能力和保水能力。叶大小通过调节光合面积和 叶表温度两条途径影响叶片的光合、呼吸速率, 与 植物相对生长速率呈显著的强相关关系(Enquist et al., 2007; Michaletz et al., 2016; Leigh et al., 2017), 进而直接或间接通过其他组织结构(如分生组织)影 响植物适合度, 例如产生更多的种子(Ackerly et al., 2000)。叶大小等性状表征了植物个体的表现，其与 
相对生长速率的关系可尺度推绎到生态系统初级生 产力(Williams et al., 2020)。

表征叶大小的重要指标之一是叶表面积, 这在 群落尺度上反映为叶面积指数 (leaf area index, LAI)。在较温暖、湿润且气候季节性较低的环境下, 叶片较大, 群落通常具有较高、较大的林冠层, 具有 较高的叶面积指数, 以增大光接受率。已有研究指 出, 叶面积指数与森林初级生产力呈正相关关系 (Gower et al., 1999; Chen et al., 2012; Reich, 2012)。 叶大小、叶面积指数与气候间的联系暗示了叶大小 可作为指示陆地生态系统初级生产力空间变化的潜 在指标。最近的研究指出, 与叶面积指数相比, 群落 内平均叶大小与生态系统初级生产力间的关系更强 (Li et al., 2020a)。限于古叶面积很难估算, 叶面积指 数并不适用于重建古初级生产力 (Dunn et al., 2015)。通过建立叶大小与初级生产力间的关系方程, 保存完好的叶化石可作为一种潜在的方法用于重建 陆生生态系统古生产力。因此, 叶大小作为重要的 叶形态指标, 可根据其与环境变量间的关系重建古 环境指标, 亦可联系植物个体尺度的光合作用和群 落尺度上的生物量积累, 为研究生态系统生产力提 供一个简单有效的功能指标。

叶大小可能与其他功能性状共变, 进而影响生 态系统功能。青藏高原高寒草甸的氮添加实验发现, 生态系统总生产力和水分利用效率随着氮添加先增 加, 随后略微降低, 这种非线性的响应与平均叶面 积的增加以及叶磷含量的降低密切相关 (Zhang et al., 2019)。此外, 叶大小可作为预测植物入侵潜 力的一个关键指标。基于新西兰 16 种本地种和 3 种入 侵种的研究发现, 在 22 个叶片结构、叶生化性状中, 唯有叶面积是入侵种与本地种显著不同的性状 (Heberling \& Mason, 2018)。入侵种与本地种的性状 组合显著不同, 前者占据叶经济型谱快速回报的一 端, 倾向于具有较大叶片、较高的资源获取和较低 的防御投入, 具有较大的最大光合速率和较高的氮 利用效率和能量利用效率(Heberling \& Mason, 2018; Mathakutha et al., 2019)。

\section{2 叶片形状的生态功能与研究进展}

广义的叶片形状(以下简称叶形)包括叶片整体 的形状以及叶尖、叶基和叶缘等部位的形态, 是表 征叶形态和植物分类的重要指标。近年来, 有研究
采用机器学习算法或数字化方法, 基于叶形和叶脉 进行植物分类(Wilf et al., 2016; 金梦然等, 2020)。狭 义的叶形通常指叶片二维表面的几何形状, 常用叶 片的长宽比和叶片最宽处的位置表征。最近基于木 兰科 9 个物种多个叶形指数的研究指出, 叶片长宽 比与叶形分形维度显著强相关, 可作为衡量叶形的 简易指标(Peijian et al., 2021)。叶形通常有两类划分 方法, 一种是定性描述, 如将叶形分为椭圆形、卵形 和倒卵形等若干类(Traiser et al., 2005; Xia et al., 2009 ) ; 另一类是依据叶长宽比分段(如, 长宽比 $<1$ 、 1-2、2-3、3-4、>4)(Jacobs, 1999; Traiser et al., 2005; Xia et al., 2009)。

叶形可影响叶片的光合面积, 较宽的叶子更能 截获足够的光。与此同时, 植物可通过改变叶片的 周长-面积比, 影响叶片边界层与外界的水气交换。 有研究指出, 被子植物的叶形变异与叶片边缘的限 制性生长和叶脉网络的整体分布有关(Pray，1955; Boyce, 2005, 2009)。据此可推测, 叶片形状的空间 变异可能受温度和水分可利用性的共同影响。在湿 热环境下, 叶片需要尽量扁平、近圆形以促进与外 界的水气交换(Hirokazu, 2005), 而在炎热、干旱的 环境下, 较厚、狭窄的叶片通常有较强的支撑结构 以抵抗枯萎(Werger \& Ellenbroek, 1978; Abrams, 1994)。

一般认为, 叶形是基因调控和自然选择双重作 用的结果(Hirokazu, 2005; Nicotra et al., 2011)。叶片 二维的扩展主要取决于原基的背腹性, 也受叶片伸 展后的环境条件 (如光照条件和重力作用) 影响 (Hirokazu，2005)。Nicotra等(2011)总结被子植物叶 形的进化和功能时指出, 叶形在不同发育阶段受多 个基因调控, 叶形变异反映了自然选择对叶片功能 的选择作用, 以使植物能更好地生长。目前已提出 多个假说解释叶形的多样性, 包括水分限制、叶片 温度调控、生物力学限制、规避植食风险和优化光 截获的适应以及响应花型选择等(Brown \& Lawton, 1991; Nicotra et al., 2011)。

叶形的变异主要反映了叶片水分供应的权衡, 这部分是因为叶形与叶脉的分布息息相关, 共同决 定了水分在叶片间的分布和运输。通过计算机模拟 3 种模式植物的叶发育, 发现叶脉网络的分布对叶形 起决定作用, 模型参数的较小改变就可产生丰富的 叶形(Runions et al., 2017)。叶形虽然也与叶片温度 
的调节有关, 但作用相对较弱, 叶温主要受叶大小、 叶厚度和叶倾角的影响(Nicotra et al., 2011)。叶形反 映了植物对生境养分的适应策略。基于富营养化湖 泊中大型沉水植物的研究表明, 环境对水生植物性 状的影响依赖于叶形, 扁平叶型沉水植物具有较高 的与光合相关的形态性状, 其性状主要受水深影响; 针叶型沉水植物则通常具有较高的化学计量性状, 性状主要受水质量影响; 与针叶型相比, 扁平叶型 植物更能改善水下的光条件和水质量(Liu et al., 2021)。此外, 叶形也与植食防御密切相关。例如, 叶形会影响象甲(Apoderus praecellens)在两种香茶 菜(Isodon umbrosus var. hakusanensis和I. trichocarpus) 上的产卵行为, 虽然两者亲缘关系相近且营养、气 味没有差别, 相比于前者叶缘深裂的叶片, 象甲更 倾向于选择后者完整(未分裂)的叶片, 因此, 叶片 分裂可视为香茶菜防御捕食的一种手段(Higuchi \& Kawakita, 2019)。

目前关于叶形的大尺度格局研究相对较少, 比 较经典的有Traiser等(2005)对欧洲当地阔叶乔木的 叶形态研究, 发现狭长的叶片(长宽比>3) 主要分布 在地中海沿岸, 圆形叶 $($ 长宽比 $<1)$ 在整个北方较多; 叶形更多地受温度影响, 且最低温的影响要显著强 于最高温。与之相对的, Glukhov和Strelnikov (2014) 发现, 15种榕属(Ficus)植物的叶形主要由夏季降水 量决定, 与年降水量的峰值、丰度以及气温的季节 性呈显著的正相关关系。 $\mathrm{Li}$ 等(2020b)在中国7种植 物的叶形研究中指出, 叶形在空间上的变异主要受 降水量影响, 而在时间上的变化更多地与年平均气 温有关。气温和降水量对叶形在时空上变异的相对 作用还有待进一步探讨。

\section{3 叶缘的生态功能及研究进展}

\section{1 叶缘的生态功能}

叶缘特征是当前叶形态研究中关注较多的植物 性状。叶缘特征一般是指叶片边缘是否具齿或是否 形成叶裂。叶齿是叶片边缘突出的结构。当突出部 分的长度小于其顶端到叶片主脉距离的 $1 / 4$ 时, 该 突出结构通常被称为叶齿(Hickey, 1973; Ellis et al., 2009), 若此长度比例大于 $1 / 4$ 则被称为叶裂(Royer et al., 2005)。叶齿形态多样, 主要可分为全缘、波 状、锯齿和牙齿等, 其中锯齿又分为锯齿状、细锯 齿、钝锯齿、圆锯齿、重锯齿等。
叶齿可以通过影响叶片的生理、生化过程调节 植物与外界生境的相互作用。目前对叶齿生态功能 的研究主要涉及以下三方面: 1)叶齿对叶片水分运 输和水分利用效率的影响(Canny, 1990; Feild et al., 2005); 2) 叶齿促进植物对低温的适应(Royer \& Wilf, 2006; Peppe et al., 2011); 3) 叶齿与叶片结构的互作 (Givnish, 1978a; Wilf, 1997)等。值得注意的是, 目前 关于叶齿生态功能的研究多是基于观察推测和实验 验证，尚缺乏机制性的解释。

首先, 叶齿影响叶片的水分利用和运输。叶齿 影响叶脉网络结构的分布(Givnish, 1978a), 齿尖通 常是叶脉在叶片边缘的分布终端(Bailey \& Sinnott, 1916)。解剖观察发现, 齿尖通常有开放气孔或排水 孔, 这些结构降低了叶片边界层的厚度, 促进叶片 与外界的水气交换, 提高水分的运输效率(Canny, 1990)。此外, 在水分充沛、地下水位浅的地方(如水 滨和湖边生境), 具齿物种占有较高的比例, 即淡水叶缘效应(Greenwood, 2005; Royer et al., 2009a)。这 是因为叶齿可以通过吐水作用缓解过高的正根压, 减轻水胁迫对植物生理活动的影响 (Feild et al., 2005)。

其次, 叶齿是叶片能量交换的热点区域, 能促 进植物对低温的适应。在生长季早期, 叶齿能增强 液流, 提高寒冷气候下叶片的光合、蒸腾速率, 促进 碳的吸收(Royer \& Wilf, 2006; Peppe et al., 2011), 弥补低温对植物光合作用的影响。Royer和Wilf (2006)通过测量不同生境下木本植物叶缘的蒸腾和 光合作用发现, 叶缘的生理活动开始较早, 尤其是 在寒冷地区, 叶缘带齿的物种在生长季早期比全缘 叶物种具有更高的光合、蒸腾作用, 说明在温度受 限时, 叶齿能够增加叶片在生长季早期的碳吸收。 在寒冷气候下, 叶齿的存在也有助于缓解冻融栓塞, 防止早春冻害(Feild et al., 2005)。因此叶齿被认为是 植物对寒冷气候的一种适应策略(Royer \& Wilf, 2006; Peppe et al., 2011)。

第三, 叶齿是叶片重要的组成结构。叶齿和叶 裂(或缺刻)可作为较薄叶片的一种保护结构, 减轻 风力下叶片被撕裂的危险 (Baker-Brosh \& Peet, 1997)。叶齿还可作为一种防御结构, 因其适口性差, 能减少植食动物的取食(Brown \& Lawton, 1991)。

叶片边缘锯齿的形成受遗传因素、环境和植物 
特性的共同调控。首先，叶齿的发育涉及多个基因 的表达调控。有研究显示, TCP转录因子在植物叶形 态多样化的过程中起到重要作用, 而E3泛素连接酶 TEAR1可通过降解转录抑制因子TIE1而增强TCP转 录因子的活动, 因此TEAR1过度表达有利于叶片边 缘完整, 破坏TEAR1及其同源基因的功能可使得叶 缘锯齿增多(Zhang et al., 2017)。此外, 叶齿发育也 受植物激素的影响。例如, 生长素浓度直接控制叶 缘锯齿的形成(李晓屿等, 2019)。第二, 叶齿的形成 受周围环境的影响。例如, Royer等(2009a)通过探讨 扰动、水分可利用性和植物生长策略等对澳大利亚 亚热带雨林中具齿植物的影响, 检验了淡水-叶缘 假说、盐胁迫假说、生活策略假说以及澳大利亚无 齿假说, 证明了区域内水分可利用性对具齿物种分 布的重要影响。第三, 叶齿的形成与植物的特性有 关。植物生活型(乔木、灌木和草本)、叶习性(常绿、 落叶)、叶厚度以及比叶面积等均影响物种的叶缘状 态(是否具齿)。多个研究发现, 落叶物种的叶片叶缘 倾向于具齿, 而常绿物种倾向于叶片全缘(Jacobs, 1999; Adams et al., 2008; Royer et al., 2012)。叶片厚 度也显著影响叶缘是否具齿, 且齿的尖锐程度受叶 片厚度调控。这是因为较薄的叶片需要更强的支撑 结构以维持其伸展, 在叶脉伸向叶缘时, 脉间的叶 片组织可能因为没有足够的结构支持而缺失, 从而 形成波状或齿状的叶缘(Givnish, 1978a)。相对应地, 厚叶能减小胞内水流所受的阻力, 有利于二级叶脉 间的均匀生长, 从而形成光滑的叶缘(Wilf, 1997)。

环境特征和植物特性对叶缘特征的影响是综合 的, 而非某一因素单独起作用。比如, 结合叶经济谱, 与常绿种相比, 落叶种的叶片寿命相对较短, 处于 快速回报的一端, 倾向于在叶片上投入较少的生物 量, 因此叶片相对较薄(Givnish, 1978a; Wright et al., 2004)。较薄的叶片倾向于具齿以增强叶片的物理支 撑, 避免在强风中撕裂(Baker-Brosh \& Peet, 1997)。 再如, 落叶性和叶齿间的强相关性可用叶片气体交 换假说解释(Royer \& Wilf, 2006)。在寒冷的气候下, 叶齿通常能增强生长季早期的气体交换, 加强碳固 定, 因此叶齿可能是落叶物种对低温的一种适应性 策略(Peppe et al., 2011; Royer et al., 2012)。Li等 (2016)在分析中国木本植物的叶缘特征时发现, 常 绿和落叶物种的叶缘特征差异在乔木中要强于灌木, 即常绿(或落叶)乔木比常绿(或落叶)灌木具有更高
比例的全缘叶(或具齿叶)物种, 这可能意味着长得 高且生活周期长的植物的叶缘状态更容易受温度影 响。需要注意的是, 叶习性对叶缘状态的影响与温 度对叶缘状态的影响共相关, 因为常绿种通常分布 在温暖的区域, 而在寒冷的区域相对较少。因此, 在 探讨叶习性对叶缘的影响时需要考虑如何控制或消 除气候的影响。

\section{2 叶缘组成的空间格局及其与气候的关系}

叶缘组成是指一定区域内全缘叶物种(或具齿 叶物种)占区域内全部物种的比例。叶缘组成与气候 间的关系一直是古生态学家研究的热点 (Wolfe, 1979; Adams et al., 2008; Peppe et al., 2011)。叶缘分 析通过研究现有植被的叶缘组成与年平均气温的关 系, 构建转换方程, 再根据叶化石重建古温度 (Bailey \& Sinnott, 1915; Wolfe, 1979, 1993)。叶缘分 析最早起源于Bailey和Sinnott在20世纪初对木本植 物区系的观察。他们发现, 热带和亚热带地区的双 子叶植物具有较高比例的全缘叶物种, 而温带地区 则具有较高比例的具齿叶物种 (Bailey \& Sinnott, 1915, 1916)。之后, Wolfe等(1979)研究了东亚和北美 植物区系的叶缘组成, 首次定量验证了叶缘组成与 年平均气温间的线性关系(图3), 并将其用于重建第 三纪气候。

此后, 叶缘组成与年平均气温间的关系被广泛 研究。多个研究表明, 区域内植物的全缘叶物种比

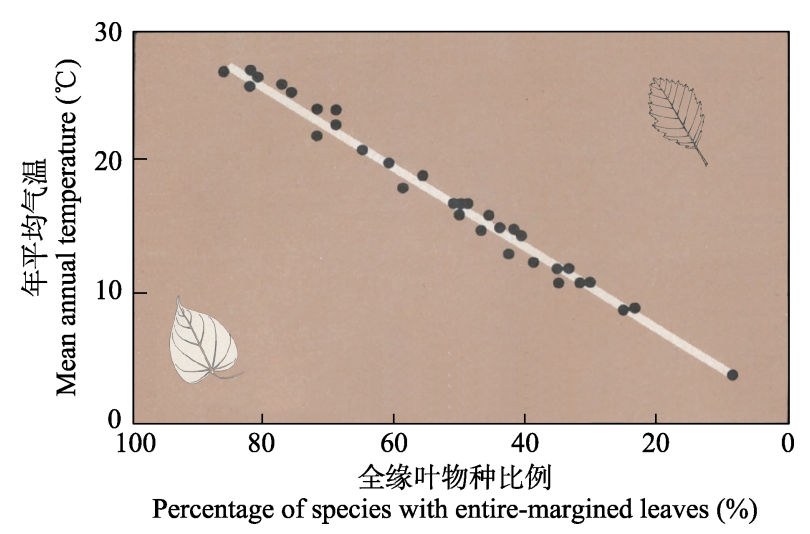

图3 东亚阔叶林中全缘叶物种比例随年平均气温升高而增 大。从左到右, 全缘叶物种降低, 具齿叶物种增多。白色实 线为回归拟合线, 决定系数高达 0.98 。改编自Wolfe等(1979), 图2-3。

Fig. 3 Percentage of species with entire-margined leaves increased with mean annual temperature in broad-leaved forests of east Asia. From left to right, the percentage of species with entire leaves increased while those with teethed leaves decreased. Fitted regression line was showed in white with $R^{2}=$ 0.98. Modified from Fig. 2 \& 3 in Wolfe et al. (1979). 
例与当地的年平均气温存在显著的线性关系, 可用 于重建古温度(Gregory-Wodzicki, 2000; Royer et al., 2005, 2009a; Royer \& Wilf, 2006; Peppe et al., 2011; Chen et al., 2014)。基于这一关系, 人们已在北美 (Wolfe, 1979, 1995; Dolph \& Dilcher, 1980; Wilf et al., 1998; Adams et al., 2008)、南美(Kowalski, 2002)、 欧洲南部(Traiser et al., 2005)、非洲热带地区(Jacobs, 1999)、东亚(Wolfe, 1979; Su et al., 2010; Chen et al., 2014)、澳大利亚(Greenwood et al., 2004; Greenwood, 2005), 乃至全球(Peppe et al., 2011; Royer et al., 2012) 建立多个温度重建的转换方程。然而, 不同区域构 建的转换方程在模型拟合度和回归斜率上存在显著 的差异(Greenwood et al., 2004; Adams et al., 2008; Peppe et al., 2011), 限制了转换模型的普适性与外 推。此外, 目前基于大尺度连续分布数据的定量叶 缘分析还很欠缺(Li et al., 2016), 叶缘组成对温度 的响应及其潜在的生态影响因子尚需进一步研究。

以往的叶缘分析主要关注叶缘组成与温度间的 关系(Gregory-Wodzicki, 2000; Royer et al., 2005, 2009a; Royer \& Wilf, 2006; Peppe et al., 2011; Chen et al., 2014)。基于叶齿的生态学功能, 叶缘状态与 水分运输和利用率密切相关。在水分充足的湿润地 区, 叶缘可能更多地响应温度的变化(Adams et al., 2008), 因而全缘叶物种比例与温度表现出很强的 相关性。与之相对的, 干旱地区的植物会采取一些 适应性策略以应对缺水, 例如调整叶大小和叶型等 (Parkhurst \& Loucks, 1972; Givnish \& Vermeij, 1976), 从而降低温度对叶缘的影响。Wolfe (1993)在分析全 球样点的叶缘时指出, 相对于来自湿润地区的样点, 来自干旱、寒冷地区以及高原地区的样点更多地表 现为离群值(outliers), 即这些地区的全缘叶物种比 例明显不同于其他地区。已有研究探讨了叶缘组成 与降水量间的关系(Wiemann et al., 1998; Wilf et al., 1998; Peppe et al., 2011; Moles et al., 2014)。定量化 年平均气温和降水量对叶缘的相对影响, 有助于评 估叶缘分析用于古温度重建的不确定性。

叶缘组成与气候间的关系也受植物生活型的影 响。有研究指出, 木本植物、草本植物和偋类植物 在叶缘组成与温度间的关系上存在显著差异: 木本 植物由于其生活周期较长, 叶缘状态受周围环境的 影响较大(Traiser et al., 2005); 而草本和蒝类植物因 其茎叶独特的导水方式和叶脉分布类型, 叶缘受外
界气候的影响较小(Royer et al., 2012; Peppe et al., 2014)。此外，乔灌草也具有不同的叶缘-温度关系， 即乔木的叶缘状态对温度响应最敏感，灌木居中， 草本最弱(Royer et al., 2012)。目前大尺度的叶缘分 析多以双子叶木本植物或被子植物为研究对象, 如 对欧洲阔叶树的叶缘研究(Traiser et al., 2005)、对全 球双子叶被子植物的研究(Royer et al., 2012)等; 部 分研究以双子叶乔木为研究对象, 如对北美(Adams et al., 2008)和对中国湿润地区(Chen et al., 2014)双 子叶乔木的叶缘研究, 均将大灌木同乔木一起纳入 分析。值得注意的是, 以往研究较少考虑叶缘组成 与气候间的关系在不同生活型(如乔木与灌木)间的 差异。 $\mathrm{Li}$ 等(2016)研究指出, 在中国干旱地区, 各生 活型的全缘叶物种比例与温度均具有较弱的相关性, 降水量增多并不能加强灌木的叶缘与温度间的关系, 但湿润地区的乔木叶缘与温度呈显著强相关关系。

与叶缘有关的叶性状具有很强的谱系保守性 (Jordan, 1997), 说明植物叶缘是否具齿是一种谱系 保守的性状，进化历史可能影响叶缘分布的格局 (Dolph \& Dilcher, 1980; Ackerly, 2004; Schmerler et al., 2012)及其与温度间的关系(Jordan，1997; Little et al., 2010; Jordan, 2011)。Little等(2010)根据北 美17个样点的叶缘数据发现, 叶缘状态存在很显著 的谱系信号, 进化历史影响叶缘组成与年平均气温 间的关系。叶缘分析的前提假设是叶缘与气温间的 关系在地质历史上保持稳定，这一假设可能并不准 确。有学者认为现代叶缘与气温间的关系可能是第 四纪冰期以来环境篮选作用的结果, 并推测地质年 代越久远，叶缘分析的不确定性越大(Jordan，1997， 2011)。 Li等(2016)用中国木本植物进一步验证了宏 观进化历史对叶缘-温度间关系的影响, 发现科年 龄显著影响叶缘组成与年平均气温间的关系, 尤其 是中国特有种, 叶缘与气温间关系随着科年龄的增 加而变弱, 叶缘分析在古老科中的不确定性变大。

植物叶缘组成与气候间的关系还受其他因素的 影响, 例如区系内物种组成、地形异质性、土壤性 质以及系统发育历史等(Wilf, 1997; Adams et al., 2008; Peppe et al., 2011)。对北美乔木的叶缘分析发 现，全缘叶物种比例与年平均气温呈非线性关系， 且北美西海岸乔木的叶缘组成与温度并没有显著的 相关性, 这可能与该区域地形复杂或者植物区系组 成不完整有关(Adams et al., 2008)。对澳大利亚的叶 
缘分析发现, 澳大利亚与其他区域相比具有较高比 例的全缘叶物种 (即“澳大利亚无齿”假说), 且其叶 缘组成与温度间的关系也比其他地区弱 (Greenwood, 1992; Greenwood et al., 2004), 这可能是由于澳大 利亚独特的地质历史和进化历史。由于叶缘与气候 的关系受多因素的影响且存在区域差异, 因而, 基 于不同区域建立的转换模型可能并不具有广泛适用 性。这些发现为叶缘分析校准古温度带来一定的不 确定性。

在叶缘分析中, 早期研究所选用的叶缘指标通 常为区域内叶片具齿(或全缘)的物种比例, 齿的数 目与大小、叶裂程度和叶齿形状相关的研究较少。 近年来随着扫描和分析技术的进步, 关于齿的类 型、数目和大小的研究增多 (Jacobs, 1999; Royer et al., 2005; Peppe et al., 2011)。叶齿数目和大小均与 年平均气温呈负相关关系, 在寒冷的环境下, 叶片 通常具有更多、更大的齿(Royer et al., 2005; Peppe et al., 2011)。此外, 对Acer rubrum的研究发现, 叶齿数 目和叶裂程度对气候变化的响应非常敏感(Royer et al., 2009b)。在未来研究中, 应进一步突破叶缘分 析仅聚焦于全缘叶物种比例这一单一指标的限制, 通过结合运用叶齿类型、数目、大小等定量指标, 综 合分析叶缘特征与气候间的关系, 为古气候重建提 供更准确的方法。

\section{4 叶型的生态功能及其空间分布}

叶型有单叶和复叶之分。单叶只有一个叶片和 叶柄, 复叶则有多个小叶沿叶轴分布, 且小叶与叶 轴间通常连有小叶柄。一般认为复叶起源于单叶, 单叶的叶缘出现缺刻并增大, 先形成叶裂, 之后缺 刻加大逐渐进化至叶片全裂, 当裂片与主叶轴之间 出现小叶柄或者小叶与叶轴间的关节明显时, 复叶 形成。在叶型的发育上, 复叶比单叶有着更复杂的 形态建成过程。最近的研究通过解析豆科模式植

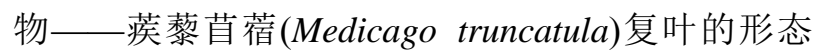
建立机制, 发现域蛋白PINNA1可独立也能与其他 蛋白协同合作, 实现对复叶中小叶数目与排列方式 的精细调控(He et al., 2020)。小叶作为复叶的一部 分, 并不是独立的单元, 复叶的小叶在某些生理功 能上类似于单叶的叶裂或叶齿(Xu et al., 2009)。

关于复叶的生态形成机制, 主要有两个假说, 分别是季节性干旱假说(Givnish, 1978b; Gates, 1980)
和快速生长适应假说(Niinemets et al., 2006; Malhado et al., 2010)。季节性干旱假说从叶片结构入手, 对比了单叶和复叶的水分利用效率, 发现与单叶相 比, 相同面积的复叶具有较大的周长面积比, 能增 强对流, 有效地散热, 从而降低叶片表面温度和蒸 腾作用, 有利于保持水分(Gates, 1980)。此外, 复叶 物种因能便捷地脱去小枝, 在面临干旱时可以快速 地减小叶面积, 有效降低蒸腾, 因此复叶物种在温 暖、干旱半干旱且强光的环境下比较常见(Givnish, 1978a; Stowe \& Brown, 1981)。快速生长适应假说则 考虑物质投入，即单、复叶的生物量分配。复叶在 支撑组织, 如叶轴、叶柄和叶脉上的投入较多 (Niinemets et al., 2006; Wu et al., 2019), 这增加了侧 向生长的投入, 但相对于永久性枝条等木质结构, 复叶的叶轴比较“廉价”, 可减少植株侧枝的生物量 分配，从而更有效地促进垂直生长(Malhado et al., 2010)。Malhado等(2010)基于亚马孙雨林137个永久 样地的研究发现, 复叶物种具有较低的木材密度和 较快的直径生长速率，从而支持了快速生长假说。 此外, 许多先锋种是复叶植物(Givnish, 1978b), 而 先锋种通常生长较快以占领新的生境, 这一现象间 接支持了快速生长假说。

目前关于叶型的研究以对比单、复叶物种的解 剖结构、生理生态等过程的差异为主，如单叶和复 叶物种在叶片解剖结构上的差异(Koch et al., 2018), 在叶片和叶柄等组织结构上的生物量分配 (Niinemets, 1998), 在叶片光合、导水率等生理活动 上的差异(Yang et al., 2019a; 赵万里等, 2019)等。赵 万里等(2019)通过对比分析豆科 11 个复叶树种和 6 个单叶树种，指出复叶树种的正午枝条水势和气孔 导度降低比例均显著高于单叶树种, 即复叶树种能 在缺水状态下快速降低气孔导度, 以降低枝条气穴 化风险。单、复叶与其他叶性状(如叶柄长度、叶面 积、比叶面积和元素含量等)的关系也是研究的热点 (Niinemets, 1998; Warman et al., 2011; Wu et al., 2019)。与单叶相比, 复叶有相对较高的水分传导率 和光合速率, 因此具有较高的资源获取能力和相对 生长速率(Wu et al., 2019; Yang et al., 2019a)。此外, 有研究指出, 复叶在耐阴性和规避植食上与单叶相 比没有显著不同(Niinemets，1998; Warman et al., 2011)。

了解复叶物种的分布及其空间变异有利于理解 
物种共存和生态位分化的机制。研究发现, 复叶乔 木倾向于出现在春夏温度较高且降水量较少的地区 (Xu et al., 2009)。在小尺度上，复叶的小叶容易脱落， 在光缝隙处较常见(Givnish, 1978b); 而在区域尺度 上, 复叶物种在热带雨林中比较常见, 且复叶出现 的频率与地形和植物习性有关(Rollet, 1990; Turner, 2001)。目前关于复叶物种分布与气候间关系的研究 相对较少。对北美乔木的研究发现, 复叶在春、夏 温度较高且降水量较低的区域比较常见(Stowe \& Brown, 1981)。

\section{5 展望}

目前, 植物叶片形态数据主要通过三种途径获 取, 一是野外直接采样, 二是来自野外或温室受控 实验, 三是从公开数据库或者已发表的植物志和其 他文献中收集。三种方法各有利弊(Wilf，1997;

Wiemann et al., 1998; Royer et al., 2005; Traiser et al., 2005; Peppe et al., 2011)。前两种方法获取的数据直 接、可靠, 且能考虑性状的种间、种内差异, 但耗时 费力, 仅限于小区域部分类群的研究, 难以拓展到 大尺度。整合数据的方法可用于大尺度的研究, 数 据获取性强, 但无法考察种内的形态变异(Wolfe et al., 1999)。此外, 整合数据受数据库中数据质量和 覆盖度的影响, 需要考虑不同来源的数据在测量方 法上的差异、对类群和区域的采样偏好以及数据库 间重复收录等问题。

目前在线公开的植物性状数据库中, TRY植物 性状数据库(TRY Plant Trait Database, https://www.trydb.org/TryWeb/Prop0.php)和BIEN植物信息与生态 网络(Botanical Information and Ecology Network, http://bien.nceas.ucsb.edu/bien/biendata/)两大集成数 据库关注度最高, 物种数和性状涵盖度广且获取方 便(Kattge et al., 2011; Maitner et al., 2018; 付全升等, 2021)。TRY数据库需要在其网页上申请, 申请时需 要提供项目主持人的基本信息、项目简介和研究摘 要。BIEN数据库可联系负责人直接提取, 也可通过 R编程软件下载, 即“BIEN”程序包(Maitner et al., 2018)。值得注意的是, TRY和BIEN等全球数据库所 收录的数据大部分来源于欧洲和南、北美洲, 亚洲 地区的数据相对较少。中国现有部分发表的数据文 章, 例如Wang 等(2018)发表的中国植物性状数据 库、Geng等(2017)发表的中国草原性状数据等。近
年来, 中国森林大样地开展了大量的调查和观测实 验, 在样地群落水平上积累了大量的植物性状数据 (详见中国森林生物多样性监测网, Chinese Forest Biodiversity Monitoring Network, http://www.cfbiodiv. org/), 也可作为很好的补充数据来源。

除上文提到的叶缘分析, 目前叶形态的研究方 法还有CLAMP (Wolfe, 1995)、数字叶相分析(Digital Leaf Physiognomy, DLP)(Huff et al., 2003; Royer et al., 2005)等。三者的对比性研究较多(Wilf, 1997; Wiemann et al., 1998; Royer et al., 2005; Peppe et al., 2011)。CLAMP是多变量分析方法, 分析多个(一般 为29或 31 个)叶形态分类变量与气候变量间的关系。 CLAMP已被广泛用于区域尺度的叶大小和叶形态 研究(Jacobs, 1999; Traiser et al., 2005; Xia et al., 2009)。DLP则是一种新电子图像方法, 更多地依赖 于电脑运算, 多选择连续的变量 (Peppe et al., 2011)。当下图像数字化技术的发展将推动CLAMP 和DLP方法的进步。

植物叶片的形态变化和空间分布是环境过滤和 生物互作共同作用的结果, 反映了植物对生境的适 应。目前的研究多聚焦在局域尺度下特定的类群, 分析叶形态的空间分布及其与环境间的关系, 并推 测叶性状分布的成因及其生态学意义, 探索植物如 何响应环境变化, 对生态系统功能有何影响。未来 主要的研究方向如下:

(1)考虑叶形态的可塑性, 获得覆盖类群和区域 无偏的性状数据是重中之重。一方面, 整合现有的 性状数据资源, 规范、简约化性状共享平台 (Gallagher et al., 2020); 另一方面, 评估性状缺失的 类群和区域分布, 进行野外采样或者用统计方法对 性状进行填补(gap filling)。近年来遥感技术的发展, 尤其是高光谱遥感技术，可大批量获得高分辨率的 连续性状数据(Jetz et al., 2016)。已有研究尝试联合 成像光谱和激光雷达等技术探索区域植被的性状变 化(Durán et al., 2019; Ma et al., 2019)。技术的难点 之一是如何从成像光谱中获取目标性状的分布。机 器学习方法的发展, 包括随机森林、神经网络和贝 叶斯分类器等, 为建立地面植物观测点和高光谱间 的关系模型提供了方法支持。

(2)叶形态在不同时间尺度上的变化及其所代 表的适应策略和生态效应有待进一步研究。目前叶 形态变化的研究多基于空间尺度，以空间置换时间， 
推测气候变化下植物性状变化对生态系统功能的潜 在影响, 存在较大的不确定性。借助多年积累的标 本数据或者设计长期的观测实验有助于从相对短时 间尺度上准确理解植物叶形态对气候变化的响应 (Li et al., 2020b; Zhu et al., 2020)。如何在更长时间 尺度上探讨叶形态的进化适应性还有待进一步研 究。目前进化和生态因素对叶形态变异的相对作用 尚不明确, 在宏观尺度上, 叶形态的变异可能更多 地受生态因素的影响。

(3)从叶片到个体再到生态系统, 探索不同尺度 上叶性状对环境变化的响应及其与生态系统功能间 的关系是研究热点。虽然叶形态间关系及其与环境 间关系的研究很多, 但因研究对象或研究尺度的不 同, 所得结论不尽相同(Messier et al., 2017)。如何减 少研究尺度的影响以及尺度推绎是以后研究的关 键。不同叶性状对生态系统功能的作用是否具有尺 度一致性以及各叶性状在不同尺度上的相对贡献如 何还有待进一步探讨。

\section{参考文献}

Abrams MD (1994). Genotypic and phenotypic variation as stress adaptations in temperate tree species: a review of several case studies. Tree Physiology, 14, 833-842.

Ackerly DD (2004). Adaptation, niche conservatism, and convergence: comparative studies of leaf evolution in the California chaparral. The American Naturalist, 163, 654-671.

Ackerly DD, Dudley SA, Sultan SE, Schmitt J, Coleman JS, Linder CR, Sandquist DR, Geber MA, Evans AS, Dawson TE, Lechowicz MJ (2000). The evolution of plant ecophysiological traits: recent advances and future directions. Bioscience, 50, 979-995.

Ackerly DD, Knight CA, Weiss SB, Barton K, Starmer KP (2002). Leaf size, specific leaf area and microhabitat distribution of chaparral woody plants: contrasting patterns in species level and community level analyses. Oecologia, 130, 449-457.

Adams JM, Green WA, Zhang Y (2008). Leaf margins and temperature in the North American flora: recalibrating the paleoclimatic thermometer. Global and Planetary Change, 60, 523-534.

Ali S, Khan N, Xie LN (2020). Molecular and hormonal regulation of leaf morphogenesis in Arabidopsis. International Journal of Molecular Sciences, 21, 6132. DOI: 10.3390/ ijms21145132.

Bailey IW, Sinnott EW (1915). A botanical index or cretaceous and tertiary climate. Science, 41, 831-834.

Bailey IW, Sinnott EW (1916). The climatic distribution of certain types of Angiosperm leaves. American Journal of
Botany, 3, 24-39.

Baker-Brosh KF, Peet RK (1997). The ecological significance of lobed and toothed leaves in temperate forest trees. Ecology, 78, 1250-1255.

Boyce CK (2005). Patterns of segregation and convergence in the evolution of fern and seed plant leaf morphologies. Paleobiology, 31, 117-140.

Boyce CK (2009). Seeing the forest with the leaves - Clues to canopy placement from leaf fossil size and venation characteristics. Geobiology, 7, 192-199.

Brown VK, Lawton JH (1991). Herbivory and the evolution of leaf size and shape. Philosophical Transactions of the Royal Society of London Series B: Biological Sciences, 333, 265-272.

Canny MJ (1990). Fine veins of dicotyledon leaves as sites for enrichment of solutes of the xylem sap. New Phytologist, 115, 511-516.

Chapin FS, Autumn K, Pugnaire F (1993). Evolution of suites of traits in response to environmental stress. The American Naturalist, 142, S78-S92.

Chen JM, Mo G, Pisek J, Liu J, Deng F, Ishizawa M, Chan D (2012). Effects of foliage clumping on the estimation of global terrestrial gross primary productivity. Global Biogeochemical Cycles, 26, GB1019. DOI: 10.1029/ 2010GB003996.

Chen W, Su T, Adams JM, Jacques FMB, Ferguson DK, Zhou ZK (2014). Large-scale dataset from China gives new insights into leaf margin-temperature relationships. $\mathrm{Pa}$ laeogeography, Palaeoclimatology, Palaeoecology, 402, 73-80.

Conesa MÀ, Muir CD, Molins A, Galmés J (2020). Stomatal anatomy coordinates leaf size with Rubisco kinetics in the Balearic Limonium. AoB PLANTS, 12, plz050. DOI: 10.1093/aobpla/plz050.

Corner EJ (1949). The durian theory or the origin of the modern tree. Annals of Botany, 13, 367-414.

Cristofori V, Rouphael Y, Gyves EMD, Bignami C (2007). A simple model for estimating leaf area of hazelnut from linear measurements. Scientia Horticulturae, 113, 221-225.

Dolph GE, Dilcher DL (1980). Variation in leaf size with respect to climate in the tropics of the Western Hemisphere. Bulletin of the Torrey Botanical Club, 107, 154-162.

Dunn RE, Strömberg CAE, Madden RH, Kohn MJ, Carlini AA (2015). Linked canopy, climate, and faunal change in the Cenozoic of Patagonia. Science, 347, 258-261.

Durán SM, Martin RE, Díaz S, Maitner BS, Malhi Y, Salinas N, Shenkin A, Silman MR, Wieczynski DJ, Asner GP, Bentley LP, Savage VM, Enquist BJ (2019). Informing trait-based ecology by assessing remotely sensed functional diversity across a broad tropical temperature gradient. Science Advances, 5, eaaw8114. DOI: 10.1126/sciadv. aaw8114.

www.plant-ecology.com 
Duursma RA, Falster DS, Valladares F, Sterck FJ, Pearcy RW, Lusk CH, Sendall KM, Nordenstahl M, Houter NC, Atwell BJ, Kelly N, Kelly JWG, Liberloo M, Tissue DT, Medlyn BE, Ellsworth DS (2012). Light interception efficiency explained by two simple variables: a test using a diversity of small- to medium-sized woody plants. New Phytologist, 193, 397-408.

Eisenring M, Unsicker SB, Lindroth RL (2021). Spatial, genetic and biotic factors shape within-crown leaf trait variation and herbivore performance in a foundation tree species. Functional Ecology, 35, 54-66.

Ellis B, Daly DC, Hickey LJ, Johnson KR, Mitchell JD, Wilf P, Wing SL (2009). Manual of Leaf Architecture. Cornell University Press, Ithaca, USA.

Enquist BJ, Kerkhoff AJ, Stark SC, Swenson NG, McCarthy MC, Price CA (2007). A general integrative model for scaling plant growth, carbon flux, and functional trait spectra. Nature, 449, 218-222.

Falster DS, Westoby M (2003). Leaf size and angle vary widely across species: What consequences for light interception? New Phytologist, 158, 509-525.

Fan X, Yan X, Qian C, Bachir DG, Yin X, Sun P, Ma XF (2020). Leaf size variations in a dominant desert shrub, Reaumuria soongarica, adapted to heterogeneous environments. Ecology and Evolution, 10, 10076-10094.

Feild TS, Sage TL, Czerniak C, Iles WJD (2005). Hydathodal leaf teeth of Chloranthus japonicus (Chloranthaceae) prevent guttation-induced flooding of the mesophyll. Plant, Cell \& Environment, 28, 1179-1190.

Fonseca CR, Overton JM, Collins B, Westoby M (2000). Shifts in trait-combinations along rainfall and phosphorus gradients. Journal of Ecology, 88, 964-977.

Franks PJ, Farquhar GD (2007). The mechanical diversity of stomata and its significance in gas-exchange control. Plant Physiology, 143, 78-87.

Fu QS, Huang XH, Shen SK, Deng T, Sun H (2021). Bibliometric analysis of the status quo of plant functional traits research based on databases across the Web of Science. Chinese Journal of Applied and Environmental Biology, 27, 228-240. [付全升, 黄先寒, 申仕康, 邓涛, 孙航 (2021). 基于数据库的植物功能性状研究现状文献计量 学分析. 应用与环境生物学报, 27, 228-240.]

Gallagher RV, Falster DS, Maitner BS, Salguero-Gómez R, Vandvik V, Pearse WD, Schneider FD, Kattge J, Poelen JH, Madin JS, Ankenbrand MJ, Penone C, Feng X, Adams VM, Alroy J, et al. (2020). Open Science principles for accelerating trait-based science across the Tree of Life. Nature Ecology \& Evolution, 4, 294-303.

Gates DM (1980). Biophysical Ecology. Springer, New York, USA.

Geng Y, Ma W, Wang L, Baumann F, Kühn P, Scholten T, He JS (2017). Linking above- and belowground traits to soil and climate variables: an integrated database on China's grassland species. Ecology, 98, 1471-1471.

Givnish T (1984). Leaf and Canopy Adaptations in Tropical Forests. Springer, Dordrecht, the Netherlands. 51-84.

Givnish TJ (1978a). Ecological aspects of plant morphology: leaf form in relation to environment. Acta Biotheoretica, 27, 83-142.

Givnish TJ (1978b). On the Adaptive Significance of Compound Leaves, With Particular Reference to Tropical Trees. Cambridge University Press, Cambridge, UK.

Givnish TJ, Vermeij GJ (1976). Sizes and shapes of liane leaves. The American Naturalist, 110, 743-778.

Glukhov AZ, Strelnikov II (2014). Lamina shape variability in species of the genus Ficus L. in different ecological conditions. Contemporary Problems of Ecology, 7, 210-220.

Gower ST, Kucharik CJ, Norman JM (1999). Direct and indirect estimation of leaf area index, fAPAR, and net primary production of terrestrial ecosystems. Remote Sensing of Environment, 70, 29-51.

Greenwood DR (1992). Taphonomic constraints on foliar physiognomic interpretations of late Cretaceous and Tertiary Paleoclimates. Review of Palaeobotany and Palynology, 71, 149-190.

Greenwood DR (2005). Leaf margin analysis: taphonomic constraints. Palaios, 20, 498-505.

Greenwood DR, Wilf P, Wing SL, Christophel DC (2004). Paleotemperature estimation using leaf-margin analysis: Is Australia different? Palaios, 19, 129-142.

Gregory-Wodzicki KM (2000). Relationships between leaf morphology and climate, Bolivia: implications for estimating paleoclimate from fossil floras. Paleobiology, 26, 668-688.

Guerin GR, Wen H, Lowe AJ (2012). Leaf morphology shift linked to climate change. Biology Letters, 8, 882-886.

He L, Liu Y, He H, Liu Y, Qi J, Zhang X, Li Y, Mao Y, Zhou S, Zheng X, Bai Q, Zhao B, Wang D, Wen J, Mysore KS, et al. (2020). A molecular framework underlying the compound leaf pattern of Medicago truncatula. Nature Plants, 6, 511-521.

He N, Liu C, Tian M, Li M, Yang H, Yu G, Guo D, Smith MD, $\mathrm{Yu}$ Q, Hou J (2018). Variation in leaf anatomical traits from tropical to cold-temperate forests and linkage to ecosystem functions. Functional Ecology, 32, 10-19.

Heberling JM, Mason NWH (2018). Are endemics functionally distinct? Leaf traits of native and exotic woody species in a New Zealand forest. PLOS ONE, 13, e0196746. DOI: 10.1371/journal.pone.0196746.

Hickey LJ (1973). Classification of the architecture of dicotyledonous leaves. American Journal of Botany, 17-33.

Higuchi Y, Kawakita A (2019). Leaf shape deters plant processing by an herbivorous weevil. Nature Plants, 5, 959-964. 
Hirokazu T (2005). Leaf shape: genetic controls and environmental factors. International Journal of Developmental Biology, 49, 547-555.

Hodgson JG, Santini BA, Montserrat Marti G, Royo Pla F, Jones G, Bogaard A, Charles M, Font X, Ater M, Taleb A, Poschlod P, Hmimsa Y, Palmer C, Wilson PJ, Band SR, et al. (2017). Trade-offs between seed and leaf size (seed-phytomer-leaf theory): functional glue linking regenerative with life history strategies ... and taxonomy with ecology? Annals of Botany, 120, 633-652.

Huang Y, Lechowicz MJ, Price CA, Li L, Wang Y, Zhou D (2016). The underlying basis for the trade-off between leaf size and leafing intensity. Functional Ecology, 30, 199-205.

Huff PM, Wilf P, Azumah EJ (2003). Digital future for paleoclimate estimation from fossil leaves? Preliminary results. Palaios, 18, 266-274.

Jacobs BF (1999). Estimation of rainfall variables from leaf characters in tropical Africa. Palaeogeography, Palaeoclimatology, Palaeoecology, 145, 231-250.

Jahdi R, Arabi M, Bussotti F (2020). Effect of environmental gradients on leaf morphological traits in the Fandoghlo forest region (NW Iran). iForest, 13, 523-530.

Jetz W, Cavender-Bares J, Pavlick R, Schimel D, Davis FW, Asner GP, Guralnick R, Kattge J, Latimer AM, Moorcroft P, Schaepman ME, Schildhauer MP, Schneider FD, Schrodt F, Stahl U, Ustin SL (2016). Monitoring plant functional diversity from space. Nature Plants, 2, 16024. DOI: 10.1038/nplants.2016.24.

Jin MR, Yuan H, Huang DZ (2020). Sdudy on digital classification of plants based on characteristics of leaf shape and leaf vein. Forestry and Ecological Sciences, 35, 112-118. [金梦然, 袁航, 黄大庄 (2020). 基于叶形和叶脉特征 的植物数字化分类研究. 林业与生态科学, 35, 112-118.]

Jordan GJ (1997). Uncertainty in palaeoclimatic reconstructions based on leaf physiognomy. Australian Journal of Botany, $45,527-547$

Jordan GJ (2011). A critical framework for the assessment of biological palaeoproxies: predicting past climate and levels of atmospheric $\mathrm{CO}_{2}$ from fossil leaves. New Phytologist, 192, 29-44.

Kattge J, Diaz S, Lavorel S, Prentice C, Leadley P, Boenisch G, Garnier E, Westoby M, Reich PB, Wright IJ, Cornelissen JHC, Violle C, Harrison SP, van Bodegom PM, Reichstein $\mathrm{M}$, et al. (2011). TRY - A global database of plant traits. Global Change Biology, 17, 2905-2935.

Kawai K, Okada N (2016). How are leaf mechanical properties and water-use traits coordinated by vein traits? A case study in Fagaceae. Functional Ecology, 30, 527-536.

Kleiman D, Aarssen LW (2007). The leaf size/number trade-off in trees. Journal of Ecology, 95, 376-382.

Koch G, Rolland G, Dauzat M, Bédiée A, Baldazzi V, Bertin N,
Guédon Y, Granier C (2018). Are compound leaves more complex than simple ones? A multi-scale analysis. Annals of Botany, 122, 1173-1185.

Kowalski EA (2002). Mean annual temperature estimation based on leaf morphology: a test from tropical South America. Palaeogeography, Palaeoclimatology, Palaeoecology, 188, 141-165.

Leigh A, Sevanto S, Close JD, Nicotra AB (2017). The influence of leaf size and shape on leaf thermal dynamics: Does theory hold up under natural conditions? Plant, Cell \& Environment, 40, 237-248.

Li FL, Bao WK (2014). Elevational trends in leaf size of Campylotropis polyantha in the arid Minjiang River valley, SW China. Journal of Arid Environments, 108, 1-9.

Li XY, Ralf MX, Shahid K, Zheng YC, Li YH, Xing Q (2019). Development of plant leaf margin: advances in research. Chinese Agricultural Science Bulletin, 35, 50-56. [李晓屿, Ralf Müller-Xing, Shahid Khan, 郑玉彩, 李玉花, 邢倩 (2019). 植物叶缘锯齿发育的研究进展. 中国农学通报, 35, 50-56.]

Li YQ, Reich PB, Schmid B, Shrestha N, Feng X, Lyu T, Maitner BS, Xu XT, Li YC, Zou DT, Tan ZH, Su XY, Tang ZY, Guo QH, Feng XJ, et al. (2020a). Leaf size of woody dicots predicts ecosystem primary productivity. Ecology Letters, 23, 1003-1013.

Li YQ, Wang ZH, Xu XT, Han WX, Wang QG, Zou DT (2016). Leaf margin analysis of Chinese woody plants and the constraints on its application to palaeoclimatic reconstruction. Global Ecology and Biogeography, 25, 1401-1415.

Li YQ, Zou DT, Shrestha N, Xu XT, Wang QG, Jia W, Wang ZH (2020b). Spatiotemporal variation in leaf size and shape in response to climate. Journal of Plant Ecology, 13, 87-96.

Little SA, Kembel SW, Wilf P (2010). Paleotemperature proxies from leaf fossils reinterpreted in light of evolutionary history. PLOS ONE, 5, e15161. DOI: 10.1371/journal. pone.0015161.

Liu H, Liu G, Xing W (2021). Functional traits of submerged macrophytes in eutrophic shallow lakes affect their ecological functions. Science of the Total Environment, 760, 143332. DOI: 10.1016/j.scitotenv.2020.143332.

Liu WS, Zheng L, Qi DH (2020). Variation in leaf traits at different altitudes reflects the adaptive strategy of plants to environmental changes. Ecology and Evolution, 10, 8166-8175

Lusk CH, Grierson ERP, Laughlin DC (2019). Large leaves in warm, moist environments confer an advantage in seedling light interception efficiency. New Phytologist, 223, 13191327.

Ma X, Mahecha MD, Migliavacca M, van der Plas F, Benavides R, Ratcliffe S, Kattge J, Richter R, Musavi T, Baeten

www.plant-ecology.com 
Baeten L, Barnoaiea I, Bohn FJ, Bouriaud O, Bussotti F, Coppi A, et al. (2019). Inferring plant functional diversity from space: the potential of Sentinel-2. Remote Sensing of Environment, 233, 111368. DOI: 10.1016/j.rse.2019.111368.

Maitner BS, Boyle B, Casler N, Condit R, Donoghue J, Durán SM, Guaderrama D, Hinchliff CE, Jørgensen PM, Kraft NJB, McGill B, Merow C, Morueta-Holme N, Peet RK, Sandel B, et al. (2018). The bien R package: a tool to access the Botanical Information and Ecology Network (BIEN) database. Methods in Ecology and Evolution, 9, 373-379.

Malhado A, Whittaker RJ, Malhi Y, Ladle RJ, Ter Steege H, Phillips O, Aragão L, Baker TR, Arroyo L, Almeida S (2010). Are compound leaves an adaptation to seasonal drought or to rapid growth? Evidence from the Amazon rain forest. Global Ecology and Biogeography, 19, 852862.

Malhado ACM, Malhi Y, Whittaker RJ, Ladle RJ, ter Steege H, Phillips OL, Butt N, Aragao L, Quesada CA, AraujoMurakami A, Arroyo L, Peacock J, Lopez-Gonzalez G, Baker TR, Anderson LO, et al. (2009). Spatial trends in leaf size of Amazonian rainforest trees. Biogeosciences, 6, 1563-1576.

Mathakutha R, Steyn C, le Roux PC, Blom IJ, Chown SL, Daru BH, Ripley BS, Louw A, Greve M (2019). Invasive species differ in key functional traits from native and noninvasive alien plant species. Journal of Vegetation Science, 30, 994-1006.

McDonald PG, Fonseca CR, Overton JM, Westoby M (2003). Leaf-size divergence along rainfall and soil-nutrient gradients: Is the method of size reduction common among clades? Functional Ecology, 17, 50-57.

Mebrahtu T, Hanover JW, Layne DR, Flore JA (1991). Leaf temperature effects on net photosynthesis, dark respiration, and photorespiration of seedlings of black locust families with contrasting growth-rates. Canadian Journal of Forest Research, 21, 1616-1621.

Messier J, McGill BJ, Enquist BJ, Lechowicz MJ (2017). Trait variation and integration across scales: Is the leaf economic spectrum present at local scales? Ecography, 40, 685-697.

Michaletz ST, Weiser MD, McDowell NG, Zhou J, Kaspari M, Helliker BR, Enquist BJ (2016). The energetic and carbon economic origins of leaf thermoregulation. Nature Plants, 2, 16129. DOI: 10.1038/nplants.2016.129.

Milla R, Reich PB (2007). The scaling of leaf area and mass: the cost of light interception increases with leaf size. Proceedings of the Royal Society B: Biological Sciences, 274, 2109-2115.

Milla R, Reich PB (2011). Multi-trait interactions, not phylogeny, fine-tune leaf size reduction with increasing altitude. Annals of Botany, 107, 455-465.
Moles AT, Perkins SE, Laffan SW, Flores-Moreno H, Awasthy M, Tindall ML, Sack L, Pitman A, Kattge J, Aarssen LW, Anand M, Bahn M, Blonder B, Cavender-Bares J, Cornelissen JHC, et al. (2014). Which is a better predictor of plant traits: temperature or precipitation? Journal of Vegetation Science, 25, 1167-1180.

Nardini A, Unapuu-Pikas E, Savi T (2014). When smaller is better: leaf hydraulic conductance and drought vulnerability correlate to leaf size and venation density across four Coffea arabica genotypes. Functional Plant Biology, 41, 972-982.

Nicotra AB, Leigh A, Boyce CK, Jones CS, Niklas KJ, Royer DL, Tsukaya H (2011). The evolution and functional significance of leaf shape in the angiosperms. Functional Plant Biology, 38, 535-552.

Niinemets Ü (1998). Are compound-leaved woody species inherently shade-intolerant? An analysis of species ecological requirements and foliar support costs. Plant Ecology, 134, 1-11.

Niinemets Ü (2001). Global-scale climatic controls of leaf dry mass per area, density, and thickness in trees and shrubs. Ecology, 82, 453-469.

Niinemets Ü, Portsmuth A, Tena D, Tobias M, Matesanz S, Valladares F (2007). Do we underestimate the importance of leaf size in plant economics? Disproportional scaling of support costs within the spectrum of leaf physiognomy. Annals of Botany, 100, 283-303.

Niinemets Ü, Portsmuth A, Tobias M (2006). Leaf size modifies support biomass distribution among stems, petioles and mid-ribs in temperate plants. New Phytologist, 171, 91-104.

Niinemets Ü, Sparrow A, Cescatti A (2005). Light capture efficiency decreases with increasing tree age and size in the southern hemisphere gymnosperm Agathis australis. Trees, 19, 177-190.

Niklas KJ, Cobb ED, Niinemets Ü, Reich PB, Sellin A, Shipley B, Wright IJ (2007). "Diminishing returns" in the scaling of functional leaf traits across and within species groups. Proceedings of the National Academy of Sciences of the United States of America, 104, 8891-8896.

Nobel PS (2009). Physicochemical and Environmental Plant Physiology. 4th ed. Academic Press, San Diego, USA. 318-363.

Okajima Y, Taneda H, Noguchi K, Terashima I (2012). Optimum leaf size predicted by a novel leaf energy balance model incorporating dependencies of photosynthesis on light and temperature. Ecological Research, 27, 333-346.

Ordoñez JC, van Bodegom PM, Witte JPM, Wright IJ, Reich PB, Aerts R (2009). A global study of relationships between leaf traits, climate and soil measures of nutrient fertility. Global Ecology and Biogeography, 18, 137-149.

Parkhurst DF, Loucks OL (1972). Optimal leaf size in relation 
to environment. Journal of Ecology, 60, 505-537.

Peel JR, Sanchez MCM, Portillo JL, Golubov J (2017). Stomatal density, leaf area and plant size variation of Rhizophora mangle (Malpighiales:Rhizophoraceae) along a salinity gradient in the Mexican Caribbean. Revista de Biologia Tropical, 65, 701-712.

Peijian S, Kexin Y, Ülo N, Johan G (2021). Can leaf shape be represented by the ratio of leaf width to length? Evidence from nine species of Magnolia and Michelia (Magnoliaceae). Forests, 12, 41. DOI: 10.3390/f12010041.

Peppe DJ, Lemons CR, Royer DL, Wing SL, Wright IJ, Lusk $\mathrm{CH}$, Rhoden $\mathrm{CH}$ (2014). Biomechanical and leaf-climate relationships: a comparison of ferns and seed plants. American Journal of Botany, 101, 338-347.

Peppe DJ, Royer DL, Cariglino B, Oliver SY, Newman S, Leight E, Enikolopov G, Fernandez-Burgos M, Herrera F, Adams JM, Correa E, Currano ED, Erickson JM, Hinojosa LF, Hoganson JW, et al. (2011). Sensitivity of leaf size and shape to climate: global patterns and paleoclimatic applications. New Phytologist, 190, 724-739.

Poorter L, Rozendaal DM (2008). Leaf size and leaf display of thirty-eight tropical tree species. Oecologia, 158, 35-46.

Pray TR (1955). Foliar venation of angiosperms. II. Histogenesis of the venation of Liriodendron. American Journal of Botany, 42, 18-27.

Price CA, Wing S, Weitz JS (2012). Scaling and structure of dicotyledonous leaf venation networks. Ecology Letters, 15, 87-95.

Pyakurel A, Wang JR (2014). Leaf morphological and stomatal variations in paper birch populations along environmental gradients in Canada. American Journal of Plant Sciences, 5, 1508-1520.

Reich PB (2012). Key canopy traits drive forest productivity. Proceedings of the Royal Society B: Biological Sciences, 279, 2128-2134.

Reich PB, Wright IJ, Cavender-Bares J, Craine JM, Oleksyn J, Westoby M, Walters MB (2003). The evolution of plant functional variation: traits, spectra, and strategies. International Journal of Plant Sciences, 164, S143-S164.

Ren JS, Ji XY, Wang CH, Hu JJ, Nervo SE, Li JH (2020). Variation and genetic parameters of leaf morphological traits of eight families from Populus simonii $\times$ P. nigra. Forests, 11, 1319. DOI: 10.3390/f11121319.

Renaguli R, Liu SZ, Liu TQ, Chen BZ, Wang JS (2020). Survey of plant leaf area measurement methods. Anhui Agricultural Science Bulletin, 26, 22-23. [热娜古丽・热西提, 刘生智, 刘同金, 陈炳舟, 王家硕 (2020). 植物叶面积 测量方法综述. 安徽农学通报, 26, 22-23.]

Rollet B (1990). Leaf morphology//Rollet B, Högermannc, Roth I. Stratification of Tropical Forests as Seen in Leaf Structure. Springer, Dordrecht, the Netherlands. 1-75.

Rouphael Y, Mouneimne AH, Ismail A, Mendoza-De Gyves E,
Rivera CM, Colla G (2010). Modeling individual leaf area of rose (Rosa hybrida L.) based on leaf length and width measurement. Photosynthetica, 48, 9-15.

Royer DL, Kooyman RM, Little SA, Wilf P (2009a). Ecology of leaf teeth: a multi-site analysis from an Australian subtropical rainforest. American Journal of Botany, 96, 738-750.

Royer DL, Meyerson LA, Robertson KM, Adams JM (2009b). Phenotypic plasticity of leaf shape along a temperature gradient in Acer rubrum. PLOS ONE, 4, e7653. DOI: 10.1371/journal.pone.0007653.

Royer DL, Peppe DJ, Wheeler EA, Niinemets Ü (2012). Roles of climate and functional traits in controlling toothed vs. untoothed leaf margins. American Journal of Botany, 99, 915-922.

Royer DL, Wilf P (2006). Why do toothed leaves correlate with cold climates? Gas exchange at leaf margins provides new insights into a classic paleotemperature proxy. International Journal of Plant Sciences, 167, 11-18.

Royer DL, Wilf P, Janesko DA, Kowalski EA, Dilcher DL (2005). Correlations of climate and plant ecology to leaf size and shape: potential proxies for the fossil record. American Journal of Botany, 92, 1141-1151.

Runions A, Tsiantis M, Prusinkiewicz P (2017). A common developmental program can produce diverse leaf shapes. New Phytologist, 216, 401-418.

Sack L, Scoffoni C, McKown AD, Frole K, Rawls M, Havran JC, Tran H, Tran T (2012). Developmentally based scaling of leaf venation architecture explains global ecological patterns. Nature Communications, 3, 837. DOI: 10.1038/ ncomms 1835 .

Salisbury EJ, Oliver FW (1928). On the causes and ecological significance of stomatal frequency, with special reference to the woodland flora. Philosophical Transactions of the Royal Society of London: Series B, 216, 1-65.

Schellenberger Costa D, Zotz G, Hemp A, Kleyer M (2018). Trait patterns of epiphytes compared to other plant life-forms along a tropical elevation gradient. Functional Ecology, 32, 2073-2084.

Schmerler SB, Clement WL, Beaulieu JM, Chatelet DS, Sack L, Donoghue MJ, Edwards EJ (2012). Evolution of leaf form correlates with tropical-temperate transitions in Viburnum (Adoxaceae). Proceedings of the Royal Society B: Biological Sciences, 279, 3905-3913.

Schneider JV, Habersetzer J, Rabenstein R, Wesenberg J, Wesche K, Zizka G (2017). Water supply and demand remain coordinated during breakdown of the global scaling relationship between leaf size and major vein density. New Phytologist, 214, 473-486.

Schuepp PH (1993). Leaf boundary layers. New Phytologist, 125, 477-507.

Scoffoni C, Rawls M, McKown A, Cochard H, Sack L (2011).

www.plant-ecology.com 
Decline of leaf hydraulic conductance with dehydration: relationship to leaf size and venation architecture. Plant Physiology, 156, 832-843.

Smith DD, Sperry JS, Adler FR (2017). Convergence in leaf size versus twig leaf area scaling: Do plants optimize leaf area partitioning? Annals of Botany, 119, 447-456.

Stowe LG, Brown JL (1981). A geographic perspective on the ecology of compound leaves. Evolution, 35, 818-821.

Su T, Xing YW, Liu YSC, Jacques FM, Chen WY, Huang YJ, Zhou ZK (2010). Leaf margin analysis: a new equation from humid to mesic forests in China. Palaios, 25, 234-238.

Tao Q, Guo DS, Wei BY, Zhang F, Pang CX, Jiang H, Zhang JZ, Wei T, Gu HY, Qu LJ, Qin GJ (2013). The TIE1 transcriptional repressor links TCP transcription factors with TOPLESS/TOPLESS-RELATED corepressors and modulates leaf development in Arabidopsis. Plant Cell, 25, 421-437.

Terashima I, Hikosaka K (1995). Comparative ecophysiology of leaf and canopy photosynthesis. Plant, Cell \& Environment, 18, 1111-1128.

Tian M, Yu G, He N, Hou J (2016). Leaf morphological and anatomical traits from tropical to temperate coniferous forests: mechanisms and influencing factors. Scientific Reports, 6, 19703. DOI: 10.1038/srep19703.

Traiser C, Klotz S, Uhl D, Mosbrugger V (2005). Environmental signals from leaves-A physiognomic analysis of European vegetation. New Phytologist, 166, 465-484.

Turner IM (2001). The Ecology of Trees in the Tropical Rain Forest. Cambridge University Press, Cambridge, UK.

Vendramini F, Díaz S, Gurvich DE, Wilson PJ, Thompson K, Hodgson JG (2002). Leaf traits as indicators of resource-use strategy in floras with succulent species. New Phytologist, 154, 147-157.

Wang C, He J, Zhao TH, Cao Y, Wang G, Sun B, Yan X, Guo W, Li MH (2019). The smaller the leaf is, the faster the leaf water loses in a temperate forest. Frontiers in Plant Science, 10, 58. DOI: 10.3389/fpls.2019.00058.

Wang H, Harrison SP, Prentice IC, Yang Y, Bai F, Togashi HF, Wang M, Zhou S, Ni J (2018). The China Plant Trait Database: toward a comprehensive regional compilation of functional traits for land plants. Ecology, 99, 500.

Warman L, Moles AT, Edwards W (2011). Not so simple after all: searching for ecological advantages of compound leaves. Oikos, 120, 813-821.

Webb LJ (1959). A physiognomic classification of Australian rain forests. Journal of Ecology, 47, 551-570.

Werger MJA, Ellenbroek GA (1978). Leaf size and leaf consistence of a riverine forest formation along a climatic gradient. Oecologia, 34, 297-308.

Whitman T, Aarssen LW (2009). The leaf size/number trade-off in herbaceous angiosperms. Journal of Plant
Ecology, 3, 49-58.

Wiemann MC, Manchester SR, Dilcher DL, Hinojosa LF, Wheeler EA (1998). Estimation of temperature and precipitation from morphological characters of dicotyledonous leaves. American Journal of Botany, 85, 1796-1802.

Wilf P (1997). When are leaves good thermometers? A new case for leaf margin analysis. Paleobiology, 23, 373-390.

Wilf P, Wing SL, Greenwood DR, Greenwood CL (1998). Using fossil leaves as paleoprecipitation indicators: an Eocene example. Geology, 26, 203-206.

Wilf P, Zhang SP, Chikkerur S, Little SA, Wing SL, Serre T (2016). Computer vision cracks the leaf code. Proceedings of the National Academy of Sciences of the United States of America, 113, 3305-3310.

Williams LJ, Cavender-Bares J, Paquette A, Messier C, Reich PB (2020). Light mediates the relationship between community diversity and trait plasticity in functionally and phylogenetically diverse tree mixtures. Journal of Ecology, 108, 1617-1634.

Wolfe JA (1979). Temperature Parameters of Humid to Mesic Forests of Eastern Asia and Relation to Forests of Other Regions of the Northern Hemisphere and Australasia: Analysis of Temperature Data from More Than 400 Stations in Eastern Asia. United States Department Printing Office, Washington D.C.

Wolfe JA (1993). A Method of Obtaining Climatic Parameters from Leaf Assemblages. United States Government Printing Office, Washington D.C.

Wolfe JA (1995). Paleoclimatic estimates from Tertiary leaf assemblages. Annual Review of Earth and Planetary Sciences, 23, 119-142.

Wolfe JA, Uemura K, Wilf P, Wing SL, Greenwood DR, Greenwood CL (1999). Using fossil leaves as paleoprecipitation indicators: an Eocene example: comment and reply. Geology, 27, 91-92.

Wright IJ, Dong N, Maire V, Prentice IC, Westoby M, Díaz S, Gallagher RV, Jacobs BF, Kooyman R, Law EA, Leishman MR, Niinemets Ü, Reich PB, Sack L, Villar R, et al. (2017). Global climatic drivers of leaf size. Science, 357, 917-921.

Wright IJ, Reich PB, Westoby M, Ackerly DD, Baruch Z, Bongers F, Cavender-Bares J, Chapin T, Cornelissen JHC, Diemer M, Flexas J, Garnier E, Groom PK, Gulias J, Hikosaka K, et al. (2004). The worldwide leaf economics spectrum. Nature, 428, 821-827.

Wu B, Liu J, Jiang K, Zhou J, Wang C (2019). Differences in leaf functional traits between simple and compound leaves of Canavalia maritime. Polish Journal of Environmental Studies, 28, 1425-1432.

Xia K, Su T, Liu YS, Xing Y-W, Jacques FMB, Zhou ZK (2009). Quantitative climate reconstructions of the late Miocene Xiaolongtan megaflora from Yunnan, southwest 
China. Palaeogeography, Palaeoclimatology, Palaeoecology, 276, 80-86.

Xu F, Guo W, Xu W, Wei Y, Wang R (2009). Leaf morphology correlates with water and light availability: What consequences for simple and compound leaves? Progress in Natural Science, 19, 1789-1798.

Yang D, Niklas KJ, Xiang S, Sun S (2010). Size-dependent leaf area ratio in plant twigs: implication for leaf size optimization. Annals of Botany, 105, 71-77.

Yang D, Zhang YJ, Song J, Niu CY, Hao GY (2019a). Compound leaves are associated with high hydraulic conductance and photosynthetic capacity: evidence from trees in Northeast China. Tree Physiology, 39, 729-739.

Yang Y, Zhao J, Zhao P, Wang H, Wang B, Su S, Li M, Wang L, Zhu Q, Pang Z, Peng C (2019b). Trait-based climate change predictions of vegetation sensitivity and distribution in China. Frontiers in Plant Science, 10, 908. DOI: 10.3389/fpls.2019.00908.

Yang Y, Zhu Q, Peng C, Wang H, Xue W, Lin G, Wen Z, Chang J, Wang M, Liu G, Li S (2016). A novel approach for modelling vegetation distributions and analysing vegetation sensitivity through trait-climate relationships in China. Scientific Reports, 6, 24110. DOI: 10.1038/ srep24110.
Yates MJ, Anthony Verboom G, Rebelo AG, Cramer MD (2010). Ecophysiological significance of leaf size variation in Proteaceae from the Cape Floristic Region. Functional Ecology, 24, 485-492.

Zhang D, Peng Y, Li F, Yang G, Wang J, Yu J, Zhou G, Yang $Y$ (2019). Trait identity and functional diversity co-drive response of ecosystem productivity to nitrogen enrichment. Journal of Ecology, 107, 2402-2414.

Zhang JZ, Wei BY, Yuan RR, Wang JH, Ding MX, Chen ZY, Yu H, Qin GJ (2017). The Arabidopsis RING-Type E3 ligase TEAR1 controls leaf development by targeting the TIE1 transcriptional repressor for degradation. The Plant Cell, 29, 243-259.

Zhao WL, Zhang JL, Zhang YJ, Cao KF (2019). Analysis of photosynthesis-water relationship between simple- and compound-leafed leguminous trees. Plant Science Journal, 37, 628-636. [赵万里, 张教林, 章永江, 曹坤芳 (2019). 豆科复叶和单叶树种的光合-水分关系分析. 植物科学 学报, 37, 628-636.]

Zhu J, Zhang Y, Yang X, Chen N, Li S, Wang P, Jiang L (2020). Warming alters plant phylogenetic and functional community structure. Journal of Ecology, 108, 2406-2415.

责任编委: 吕晓涛 责任编辑: 李 敏 